\title{
The Ground State Energy of a Bose Gas with Coulomb Interaction ${ }^{\star}$
}

\author{
Joseph G. Conlon
}

Department of Mathematics, University of Missouri, Columbia, MO 65211,USA

Abstract. Let $H_{N}$ be the $2 N$ particle Hamiltonian

$$
\begin{aligned}
H_{N}=\sum_{i=1}^{2 N}\left(-\Delta_{i}\right)+ & \sum_{i<j=1}^{N}\left|x_{i}-x_{j}\right|^{-1}+\sum_{i<j=1}^{N}\left|x_{i+N}-x_{j+N}\right|^{-1} \\
& -\sum_{i, j=1}^{N}\left|x_{i}-x_{j+N}\right|^{-1}
\end{aligned}
$$

where $\Delta_{i}$ is the Laplacian in the variable $x_{i} \in \mathbb{R}^{3}, 1 \leqq i \leqq 2 N$. The operator $H_{N}$ is assumed to act on wave functions $\psi\left(x_{1}, \ldots, x_{N} ; x_{N+1}, \ldots, x_{2 N}\right)$ which are symmetric in the variables $\left(x_{1}, \ldots, x_{N}\right)$ and $\left(x_{N+1}, \ldots, x_{2 N}\right)$. Suppose $\psi$ is supported in a set $\Lambda^{2 N}$, where $\Lambda$ is a cube in $\mathbb{R}^{3}$. It is shown that if a normalized wave function $\psi$ can be written as a product of two wave functions

$$
\psi\left(x_{1}, \ldots, x_{N} ; x_{N+1}, \ldots, x_{2 N}\right)=\psi_{1}\left(x_{1}, \ldots, x_{N}\right) \psi_{2}\left(x_{N+1}, \ldots, x_{2 N}\right),
$$

and the density of particles in $\Lambda$ is constant, then $\left\langle\psi\left|H_{N}\right| \psi\right\rangle \geqq-C N^{7 / 5}$ for some universal constant $C$.

\section{Introduction}

In this paper we study the ground state energy of a Bose gas consisting of equal numbers of positive and negative particles interacting via a Coulomb potential. Thus, if the gas contains $2 N$ particles with the $N$ negative particles being located at positions $x_{1}, \ldots, x_{N} \in \mathbb{R}^{3}$, and the positive particles at $x_{N+1}, \ldots, x_{2 N} \in \mathbb{R}^{3}$, its Hamiltonian $H_{N}$ is given by

$H_{N}=\sum_{i=1}^{2 N}\left(-\Delta_{i}\right)+\sum_{i<j=1}^{N}\left|x_{i}-x_{j}\right|^{-1}+\sum_{i<j=1}^{N}\left|x_{i+N}-x_{j+N}\right|^{-1}-\sum_{i, j=1}^{N}\left|x_{i}-x_{j+N}\right|^{-1}$.

Here $\Delta_{i}$ denotes the Laplacian in the variable $x_{i}, 1 \leqq i \leqq 2 N$.

* Research supported by the University of Missouri Research Council, Austrian National Science Foundation and U.S. National Science Foundation, grant DMS 8401766 
We consider $H_{N}$ acting on wave functions $\psi\left(x_{1}, \ldots, x_{2 N}\right)$ in $L^{2}\left(I R^{6 N}\right)$ which are in the domain of the unique self-adjoint operator corresponding to $H_{N}$. We assume $\psi$ satisfies Bose statistics. Thus we take $\psi$ to be invariant under all permutations of the variables $\left(x_{1}, \ldots, x_{N}\right)$ and $\left(x_{N+1}, \ldots, x_{2 N}\right)$. Let $\varrho(x)$ be the one point function for $\psi$, so

$$
\varrho(x)=\sum_{i=1}^{2 N} \int|\psi|^{2}\left(x_{1}, \ldots, x_{i-1}, x, x_{i+1}, \ldots, x_{2 N}\right) d \hat{x}_{i} .
$$

We define a function $f(x)$ as $f(x)$ is the largest value $\lambda$, such that

$$
\int_{|x-y|<1 / \lambda} \varrho(y) d y \geqq 1 .
$$

The function $f(x)$ was introduced in [2]. It is an average value of the one third power of the density at $x$.

We may now state our main result as follows:

Theorem 1.1. Let $\Lambda$ be a cube in $\mathbb{R}^{3}$, and suppose that $\psi\left(x_{1}, \ldots, x_{2 N}\right)$ is supported in $\Lambda^{2 N}$. Define the density $\varrho$ by $\varrho=N / \operatorname{vol} \Lambda$. Assume that there is a universal constant $b$ such that $f(x) \leqq b \varrho^{1 / 3}$ for all $x \in \Lambda$. Then if $\psi$ factors into a product of two wave functions,

$$
\psi\left(x_{1}, \ldots, x_{2 N}\right)=\psi_{1}\left(x_{1}, \ldots, x_{N}\right) \psi_{2}\left(x_{N+1}, \ldots, x_{2 N}\right),
$$

there is a constant $C(b)$ depending only on $b$ such that

$$
\left\langle\psi, H_{N} \psi\right\rangle \geqq-C(b) N^{7 / 5} .
$$

At the end of Sect. 4 we estimate the value of the constant $C(b)$ in the asymptotic sense $N \rightarrow \infty$. We obtain the value $C(b)=6.5$.

Theorem 1.1 was motivated by the work of Dyson and Lenard [3, 4]. In particular Dyson and Lenard [4] obtained the result:

Theorem 1.2. There is a universal constant $C$ such that for all $\psi$ in the domain of $H_{N}$

$$
\left\langle\psi, H_{N} \psi\right\rangle \geqq-C N^{5 / 3} \text {. }
$$

Dyson [3] proved the following:

Theorem 1.3. There is a universal constant $C$ and wave functions $\psi_{N}$ such that

$$
\left\langle\psi_{N}, H_{N} \psi_{N}\right\rangle \leqq-C N^{7 / 5}
$$

Dyson used Bogoliubov's method $[1,10]$ to construct the wave functions $\psi_{N}$ of Theorem 1.3. Bogoliubov's method will also be the key ingredient in our proof of Theorem 1.1. Theorem 1.1 is a converse result to Theorem 1.3 but with two restrictions. These are (a) the assumption that there is no correlation between positive and negative particles, (b) that the density of the gas is constant.

Consider a wave function $\psi\left(x_{1}, \ldots, x_{N}\right)$ and let $K_{N}$ be the kinetic energy operator on $\psi$. Thus

$$
K_{N}=\sum_{i=1}^{N}\left(-\Delta_{i}\right)
$$


where $\Delta_{i}$ is the Laplacian in the $x_{i}$ variable. The one and two point functions $\varrho(x)$, $\varrho(x, y)$ for $\psi$ are given by

$$
\begin{gathered}
\varrho(x)=\sum_{i=1}^{N} \int|\psi|^{2}\left(x_{1}, \ldots, x_{i-1}, x, x_{i+1}, \ldots, x_{N}\right) d \hat{x}_{i}, \\
\varrho(\mathrm{x}, \mathrm{y})=\sum_{i \neq j} \int|\psi|^{2}\left(x_{1}, \ldots, x_{i-1}, x, x_{i+1}, \ldots, x_{j-1}, y, x_{j+1}, \ldots, x_{N}\right) d \hat{x}_{i, j} .
\end{gathered}
$$

We define the exchange energy $\mathscr{E}\left(|\psi|^{2}\right)$ by

$$
\mathscr{E}\left(|\psi|^{2}\right)=\frac{1}{2} \iint \frac{\varrho(x, y)}{|x-y|} d x d y-\frac{1}{2} \iint \frac{\varrho(x) \varrho(y)}{|x-y|} d x d y .
$$

Now observe that for a wave function $\psi$ of the form (1.4) the attractive Coulomb energy in $\left\langle\psi, H_{N} \psi\right\rangle$ occurs as

$$
-\iint \frac{\varrho_{1}(x) \varrho_{2}(y)}{|x-y|} d x d y
$$

where $\varrho_{1}(x)$ and $\varrho_{2}(y)$ are the one point functions for $\psi_{1}$ and $\psi_{2}$ respectively as defined by (1.9). The expression (1.12) is evidently bounded below by

$$
-\frac{1}{2} \iint \frac{\varrho_{1}(x) \varrho_{1}(y)}{|x-y|} d x d y-\frac{1}{2} \iint \frac{\varrho_{2}(x) \varrho_{2}(y)}{|x-y|} d x d y .
$$

It therefore follows that it is sufficient to prove Theorem 1.1 in the case $\psi_{1}=\psi_{2}$. If $\psi_{1}=\psi_{2}=\psi\left(x_{1}, \ldots, x_{N}\right)$, then the left side of (1.5) is twice the expression

$$
\left\langle\psi, K_{N} \psi\right\rangle+\mathscr{E}\left(|\psi|^{2}\right) .
$$

Observe that kinetic energy scales as length squared while Coulomb energy scales as length. We state now a scale invariant theorem which is equivalent to Theorem 1.1:

Theorem 1.4. Let $\Lambda$ be a cube in $\mathbb{R}^{3}$ and suppose that $\psi\left(x_{1}, \ldots, x_{N}\right)$ is a function supported in $\Lambda^{N}$, symmetric in the variables $\left(x_{1}, \ldots, x_{N}\right)$. Let $\varrho=N / \operatorname{Vol} \Lambda$ be the density, and assume that there is a universal constant $b$ such that $f(x) \leqq b \varrho^{1 / 3}$ for all $x \in \Lambda$. Then there is a constant $C(b)$ depending only on $b$ such that for an $y \alpha,-\infty<\alpha$ $<\infty$,

$$
\frac{N^{\alpha}}{\varrho^{1 / 3}}\left\langle\psi, K_{N} \psi\right\rangle+\mathscr{E}\left(|\psi|^{2}\right) \geqq-C(b) N^{7 / 5-\alpha} \varrho^{1 / 3} .
$$

It is evident that Theorem 1.4 implies Theorem 1.1 since for any density $\varrho$ we may always choose $\alpha$ with $\varrho^{1 / 3}=N^{\alpha}$. On the other hand for any $\alpha$, Theorem 1.1 implies that (1.15) holds when $\varrho^{1 / 3}=N^{\alpha}$. It then follows by scaling that (1.15) holds for all values of $\varrho$. Thus Theorems 1.1 and 1.4 are equivalent.

It is interesting to compare (1.15) with Foldy's $\varrho^{1 / 4}$ law [6] for Bose gases with Coulomb interaction. Foldy suggests that for a gas of constant density $\varrho$ in a box $\Lambda$

$$
\left\langle\psi, K_{N} \psi\right\rangle+\mathscr{E}\left(|\psi|^{2}\right) \geqq-C N \varrho^{1 / 4},
$$


at high density, where the lower bound on the right is actually achieved by a Bogoliubov wave function. For (1.15) and (1.16) to be consistent we take $\varrho^{1 / 3}=N^{\alpha}$, which implies

$$
N^{7 / 5}=N \varrho^{1 / 4} \Rightarrow \varrho^{1 / 4}=N^{2 / 5} \Rightarrow \alpha=\frac{8}{15} .
$$

When $\alpha>8 / 15$, the effect of the Dirichlet boundary conditions on $\Lambda$ are dominant in the energy, and so the energy is nonnegative. For $\alpha<8 / 15$ we construct in Sect. 5 a wave function achieving the lower bound in (1.15). It is clear that this wave function has no longer constant density in $\Lambda$. It is likely that it corresponds to a constant density wave function supported on a subbox of $\Lambda$ with density $\varrho^{\prime}$ given by $\varrho^{\prime 1 / 3}=N^{8 / 15-\alpha} \varrho^{1 / 3}$.

Our aim here is first to discuss Bogoliubov's method at a heuristic level and then proceed from there to a rigorous proof of Theorem 1.4.

\section{Bogoliubov's Method}

We consider $N$ Bosons in a box $Q$ with side of length $L$ which interact via a potential $\phi(x)$ which is periodic on $Q$. Thus $\phi(x)$ may be written as a Fourier series

$$
\phi(x)=\sum_{k \in Z^{3}} v(k) e^{2 \pi i k \cdot x / L} .
$$

We shall assume from here on that $\phi(x)$ is positive definite so $v(k) \geqq 0$ for all $k \in Z^{3}$.

The essence of Bogoliubov's method is to write the Hamiltonian for the $N$ Bosons in second quantised form. Thus for each $k \in Z^{3}$ we introduce the annihilation operator $a_{k}$, which satisfies the commutation relations

$$
\left[a_{k}, a_{m}^{*}\right]=\delta_{k, m}, \quad k, m \in Z^{3} .
$$

If the kinetic energy operator $K_{N}$ of (1.8) acts on wave functions $\psi\left(x_{1}, \ldots, x_{N}\right)$ periodic on $Q^{N}$, then $K_{N}$ may be written in the second quantised form as

$$
K_{N}=\frac{4 \pi^{2}}{L^{2}} \sum_{k \in Z^{3}} k^{2} a_{k}^{*} a_{k} .
$$

The number operator $n$ is defined by

$$
n=\sum_{k \in Z^{3}} a_{k}^{*} a_{k} .
$$

Evidently $\psi$ is an eigenfunction of $n$ with eigenvalue $N$.

Next we turn to the potential energy. We wish to express the exchange energy (1.11) corresponding to the potential $\phi$ in second quantised form. The first term is given by

$$
\iint \varrho(x, y) \phi(x-y) d x d y=\left\langle\psi\left|\sum_{k, n, r} v(k) a_{n+k}^{*} a_{r-k}^{*} a_{n} a_{r}\right| \psi\right\rangle .
$$

We may write the expression (2.5) more simply by defining the operator $A_{k}$ as

$$
A_{k}=\sum_{n \in Z^{3}} a_{n+k}^{*} a_{n} .
$$


Then, on using the commutation relations (2.2), we have

$$
\iint \varrho(x, y) \phi(x-y) d x d y=\sum_{k} v(k)\left[\left\langle\psi\left|A_{k}^{*} A_{k}\right| \psi\right\rangle-N\right]
$$

The second term in the exchange energy (1.11) may be written as

$$
\iint \varrho(x) \varrho(y) \phi(x-y) d x d y=\sum_{k} v(k)\left|\left\langle\psi\left|A_{k}\right| \psi\right\rangle\right|^{2} .
$$

Hence we have in all

$$
\mathscr{E}\left(|\psi|^{2}\right)=\frac{1}{2} \sum_{k} v(k)\left[\left\langle\psi\left|A_{k}^{*} A_{k}\right| \psi\right\rangle-\left|\left\langle\psi\left|A_{k}\right| \psi\right\rangle\right|^{2}-N\right]
$$

Now let us write

$$
J_{k}(\psi)=\left\langle\psi\left|A_{k}^{*} A_{k}\right| \psi\right\rangle-\left|\left\langle\psi\left|A_{k}\right| \psi\right\rangle\right|^{2}-N .
$$

It is evident by the Schwarz inequality that

$$
J_{k}(\psi) \geqq-N,
$$

and thus we obtain a lower bound on the exchange energy

$$
\mathscr{E}\left(|\psi|^{2}\right) \geqq-\frac{1}{2} N \sum_{k} v(k) \text {. }
$$

The bound (2.12) has already been obtained by Lieb [9].

We observe here that the estimate $(2.11)$ is not necessarily a good one. We can easily see that the $v(k)$ term in $(2.5)$ is nonzero only if there are particles with momentum $m$, where $|m|>|k| / 2$. The same is true of the $v(k)$ term in (2.8). Hence if the estimate (2.11) is to be good there must exist particles with kinetic energy at least $|k|^{2} / 4$. Bogoliubov's method shows us how to construct wave functions $\psi$ for which (2.11) is a good estimate but at the least cost of kinetic energy.

In Bogoliubov's method we assume that most particles are in the zero momentum state. Thus we may approximate $A_{k}$ by

$$
A_{k} \simeq N^{1 / 2}\left[a_{k}^{*}+\underline{a}_{k}\right],
$$

which in turn yields

$$
J_{k}(\psi) \simeq N\left[\left\langle\psi\left|a_{k}^{*} a_{k}+\underline{a}_{k}^{*} \underline{a}_{k}+a_{k} \underline{a}_{k}+a_{k}^{*} \underline{a}_{k}^{*}\right| \psi\right\rangle-\left|\left\langle\psi\left|a_{k}^{*}+\underline{a}_{k}\right| \psi\right\rangle\right|^{2}\right] .
$$

Now it turns out that we are not justified in assuming most particles are in the zero momentum state. However we may assume that most particles are in states $a_{m}$ with $|m| \leqq \gamma$. Thus we approximate $A_{k}$ by

$$
A_{k} \simeq \sum_{|m| \leqq \gamma} \lambda_{m}\left[a_{m+k}^{*}+a_{m-k}\right]
$$

where $\lambda_{m}^{2}$ is the number of particles in the $a_{m}$ state, $|m| \leqq \gamma$. Hence we take

$$
\sum_{|m| \leqq \gamma} \lambda_{m}^{2}=N
$$

Our first goal here is to find out, assuming the approximation (2.15), the least cost in terms of kinetic energy of constructing a state $\psi$ for which (2.11) is a good estimate. Our situation is the following: We have two sets of Boson operators $a_{i}, b_{i}$, 
$i=1, \ldots, n$, corresponding to the $a_{m+k}, a_{m-k}$ with $|m| \leqq \gamma$ in (2.15). Let $\lambda_{1}, \ldots, \lambda_{n}$ be a set of real parameters satisfying the equality

$$
\sum_{i=1}^{n} \lambda_{i}^{2}=N
$$

We define operators $S$ and $T$ by

$$
S=\sum_{i=1}^{n} \lambda_{i} a_{i} ; \quad T=\sum_{i=1}^{n} \lambda_{i} b_{i}
$$

and $J(\psi)$ by

$$
J(\psi)=\left\langle\psi\left|S^{*} S+T^{*} T+S T+S^{*} T^{*}\right| \psi\right\rangle-\left|\left\langle\psi\left|S^{*}+T\right| \psi\right\rangle\right|^{2} .
$$

We wish to find a lower bound to the expression

$$
J(\psi)+\left\langle\psi\left|\varepsilon \sum_{i=1}^{n} \lambda_{i}^{2}\left[a_{i}^{*} a_{i}+b_{i}^{*} b_{i}\right]\right| \psi\right\rangle
$$

for various values of the parameters $\lambda_{i}$ and $\varepsilon>0$. The second term in (2.20) will come in the rigorous theory from the kinetic energy.

It is possible to come up with a precise lower bound for (2.20) since we may diagonalise it by using Bogoliubov transformations. Let $\mathbf{a}=\left(a_{1}, \ldots, a_{n}\right)$ and $\mathbf{b}$ $=\left(b_{1}, \ldots, b_{n}\right)$ be the $n$ dimensional vectors made out of the $a_{i}$ and $b_{j}$. For $2 n \times 2 n$ matrices $M$ we define transformations

$$
\left[\begin{array}{c}
\mathbf{a} \\
\mathbf{b}^{*}
\end{array}\right]=M\left[\begin{array}{c}
\boldsymbol{\eta} \\
\zeta^{*}
\end{array}\right]
$$

Here $\boldsymbol{\eta}=\left(\eta_{1}, \ldots, \eta_{n}\right)$ and $\zeta=\left(\zeta_{1}, \ldots, \zeta_{n}\right)$ are vectors made out of operators. The matrix $M$ is a Bogoliubov transformation if $M$ satisfies the identity

$$
M^{*}\left[\begin{array}{cc}
I & 0 \\
0 & -I
\end{array}\right] M=\left[\begin{array}{cc}
I & 0 \\
0 & -I
\end{array}\right]
$$

where $I$ is the identity $n \times n$ matrix. If the $a_{i}$ and $b_{j}$ satisfy canonical commutation relations and $M$ is a Bogoliubov transformation, then so also do the $\eta_{i}, \zeta_{j}$.

The first term in $J(\psi)$ and the second term in (2.20) are the expected value of operators quadratic in $a_{i}, b_{j}$. We write the sum of these two terms in matrix form. Let $C=\left(c_{i j}\right)$ and $D=\left(d_{i j}\right)$ be $n \times n$ matrices defined by

$$
c_{i j}=\lambda_{i} \lambda_{j} ; \quad d_{i j}=\varepsilon \lambda_{i}^{2} \delta_{i j} .
$$

Then the sum of these two terms is given by

$$
\left\langle\psi\left|\left[\begin{array}{c}
\mathbf{a} \\
\mathbf{b}^{*}
\end{array}\right]^{*}\left[\begin{array}{cc}
C+D & C \\
C & C+D
\end{array}\right]\left[\begin{array}{c}
\mathbf{a} \\
\mathbf{b}^{*}
\end{array}\right]\right| \psi\right\rangle-\sum_{i=1}^{N} \lambda_{i}^{2}(1+\varepsilon) .
$$

Observe that since $\varepsilon>0$, the matrix

$$
\left[\begin{array}{cc}
C+D & C \\
C & C+D
\end{array}\right]
$$


is positive definite provided all the $\lambda_{i}$ are nonzero. If one of the $\lambda_{i}$ is zero then we may take a smaller value of $n$ for our problem so there is no loss of generality in assuming all the $\lambda_{i}$ nonzero. It follows then, on making this assumption, that there is a matrix $M$ which satisfies (2.22) and also diagonalises (2.25), so

$$
M^{*}\left[\begin{array}{cc}
C+D & C \\
C & C+D
\end{array}\right] M=\left[\begin{array}{cccc}
\alpha_{1} & & & \\
& \alpha_{n} & & \\
& & \beta_{1} & \\
& & & \beta_{n}
\end{array}\right] \text {. }
$$

The matrix term in (2.24) is therefore given by

$$
\begin{gathered}
\left\langle\psi\left|\sum_{i=1}^{n} \alpha_{i} \eta_{i}^{*} \eta_{i}+\sum_{i=1}^{n} \beta_{i} \zeta_{i} \zeta_{i}^{*}\right| \psi\right\rangle=\langle\psi| \sum_{i=1}^{n} \alpha_{i} \eta_{i}^{*} \eta_{i} \\
+\sum_{i=1}^{n} \beta_{i} \zeta_{i}^{*} \zeta_{i}|\psi\rangle+\sum_{i=1}^{n} \beta_{i} .
\end{gathered}
$$

Next we wish to compute the eigenvalues $\alpha_{i}, \beta_{i}$ in terms of the $\lambda_{i}$ and $\varepsilon$. These are given as the solutions $\mu$ to the eigenvalue equation

$$
\left[\begin{array}{cc}
C+D & C \\
C & C+D
\end{array}\right]\left[\begin{array}{c}
\mathbf{v} \\
\mathbf{w}
\end{array}\right]=\mu\left[\begin{array}{c}
\mathbf{v} \\
-\mathbf{w}
\end{array}\right]
$$

where $\mathbf{v}=\left(v_{1}, \ldots, v_{n}\right)$ and $\mathbf{w}=\left(w_{1}, \ldots, w_{n}\right)$ are $n$ dimensional vectors. In order for (2.22) to hold, the $\mathbf{v}$ and $\mathbf{w}$ must satisfy the normalisation condition

$$
|\mathbf{v}|^{2}-|\mathbf{w}|^{2}= \pm 1 \text {. }
$$

Now let $\lambda=\left(\lambda_{1}, \ldots, \lambda_{n}\right)$ be the $n$ dimensional vector corresponding to the $\lambda_{i}$. Then Eqs. (2.28) may be written as

$$
\begin{aligned}
& \langle\mathbf{v}+\mathbf{w}, \lambda\rangle \lambda+(D-\mu) \mathbf{v}=0, \\
& \langle\mathbf{v}+\mathbf{w}, \boldsymbol{\lambda}\rangle \boldsymbol{\lambda}+(D+\mu) \mathbf{w}=0 .
\end{aligned}
$$

It follows that there are constants $x$ and $y$ such that

$$
(D-\mu) \mathbf{v}=x \lambda ; \quad(D+\mu) \mathbf{w}=y \lambda .
$$

Thus, if we assume that $(D \pm \mu)$ are invertible, it follows from (2.30) and (2.31) that

$$
\begin{aligned}
& x\left[1+\left\langle(D-\mu)^{-1} \lambda, \lambda\right\rangle\right]+y\left\langle(D+\mu)^{-1} \lambda, \lambda\right\rangle=0, \\
& x\left\langle(D-\mu)^{-1} \lambda, \lambda\right\rangle+y\left[1+\left\langle(D+\mu)^{-1} \lambda, \lambda\right\rangle\right]=0 .
\end{aligned}
$$

For (2.32) to have a nonzero solution $(x, y)$ we must have

$$
\left\langle(D-\mu)^{-1} \lambda, \lambda\right\rangle+\left\langle(D+\mu)^{-1} \lambda, \lambda\right\rangle+1=0 .
$$

We may write (2.33) in terms of the $\lambda_{i}, \varepsilon$ as

$$
\sum_{i=1}^{n} \frac{\lambda_{i}^{2}}{\varepsilon \lambda_{i}^{2}-\mu}+\sum_{i=1}^{n} \frac{\lambda_{i}^{2}}{\varepsilon \lambda_{i}^{2}+\mu}+1=0
$$

Suppose the $\lambda_{i}^{2}$ are all different with

$$
0<\lambda_{1}^{2}<\lambda_{2}^{2}<\ldots<\lambda_{n}^{2} .
$$


Then it is clear that there are zeros $\beta_{1}, \ldots, \beta_{n}$ of $(2.34)$ with

$$
\varepsilon \lambda_{1}^{2}<\beta_{1}<\varepsilon \lambda_{2}^{2}<\beta_{2}<\varepsilon \lambda_{3}^{2}<\ldots<\varepsilon \lambda_{n}^{2}<\beta_{n} .
$$

These are the $\beta_{i}$ which occur in (2.27). We may take the $\alpha_{i}$ in (2.27) as $\alpha_{i}=\beta_{i}$, $i=1, \ldots, n$, and hence we have defined the $\alpha_{i}$ and $\beta_{i}$ in terms of the $\lambda_{i}, \varepsilon$. In the case where $\lambda_{i}^{2}=\lambda_{i+1}^{2}$, then $\beta_{i}=\varepsilon \lambda_{i}^{2}$.

We wish now to show that

$$
\left\langle\psi\left|\sum_{i=1}^{n} \alpha_{i} \eta_{i}^{*} \eta_{i}+\sum_{i=1}^{n} \beta_{i} \zeta_{i}^{*} \zeta_{i}\right| \psi\right\rangle \geqq\left|\left\langle\psi\left|S^{*}+T\right| \psi\right\rangle\right|^{2} .
$$

It will follow then from (2.37) that (2.20) is bounded below by

$$
\sum_{i=1}^{n} \beta_{i}-\sum_{i=1}^{n} \lambda_{i}^{2}(1+\varepsilon) \text {. }
$$

To prove (2.37) we first observe from (2.21) that

$$
S+T^{*}=[\lambda, \lambda]\left[\begin{array}{c}
\mathbf{a} \\
\mathbf{b}^{*}
\end{array}\right]=[\lambda, \lambda] M\left[\begin{array}{c}
\mathbf{\eta} \\
\zeta^{*}
\end{array}\right] .
$$

It is evident from (2.28) that the matrix $M$, which is made up of the $2 n$ linearly independent solution of (2.28), has the form

$$
M=\left[\begin{array}{cc}
V & W \\
W & V
\end{array}\right],
$$

where $V$ and $W$ are $n \times n$ matrices. Hence (2.39) yields

$$
S+T^{*}=\lambda(V+W) \boldsymbol{\eta}+\lambda(V+W) \zeta^{*} .
$$

It follows then from the Schwarz inequality that

$$
\begin{aligned}
& \left|\left\langle\psi\left|S^{*}+T\right| \psi\right\rangle\right|^{2}=\left|\left\langle\psi\left|S+T^{*}\right| \psi\right\rangle\right|^{2} \\
& \quad \leqq 2\left\langle\psi\left|\boldsymbol{\eta}^{*}(V+W)^{*} C(V+W) \boldsymbol{\eta}\right| \psi\right\rangle+2\left\langle\psi\left|\zeta^{*}(V+W)^{*} C(V+W) \zeta\right| \psi\right\rangle .
\end{aligned}
$$

It is clear that in terms of quadratic forms we have the inequality

$$
\left[\begin{array}{ll}
C & C \\
C & C
\end{array}\right] \leqq\left[\begin{array}{cc}
C+D & C \\
C & C+D
\end{array}\right]
$$

On applying the transformation $M$ to the matrices in (2.43) this becomes

$$
\left[\begin{array}{cc}
A & A \\
A & A
\end{array}\right] \leqq\left[\begin{array}{llll}
\alpha_{1} & & & \\
& \alpha_{n} & & \\
& & \beta_{1} & \\
& & & \beta_{n}
\end{array}\right],
$$

where $A$ is the matrix

$$
A=(V+W)^{*} C(V+W) \text {. }
$$


By applying (2.44) to vectors of the form $[\mathbf{u}, \mathbf{u}]$ where $\mathbf{u}$ is $n$ dimensional, and noting that $\alpha_{i}=\beta_{i}, i=1, \ldots, n$, we conclude from (2.44) that

$$
2 A \leqq\left[\begin{array}{ll}
\alpha_{1} & \\
& \alpha_{n}
\end{array}\right] .
$$

The result (2.37) follows from (2.42) and (2.46).

We turn now to the expression (2.38). It is clear from (2.36) that we have

$$
J(\psi) \geqq-N,
$$

which corresponds to (2.11). We improve (2.47) by making better estimates on the $\beta_{i}$ than in (2.36). We have the following:

Lemma 2.1. For fixed $n, N$, $\varepsilon$, there is the inequality

$$
\sum_{i=1}^{n} \beta_{i}-\sum_{i=1}^{n} \lambda_{i}^{2}(1+\varepsilon) \geqq N\left[n^{-1}\left(\varepsilon^{2}+2 \varepsilon n\right)^{1 / 2}-\varepsilon / n-1\right]
$$

and equality is obtained if all the $\lambda_{i}^{2}$ are equal.

Proof. We define $g(\mu)$ by

$$
g(\mu)=\sum_{i=1}^{n} \frac{\lambda_{i}^{2}}{\varepsilon \lambda_{i}^{2}-\mu},
$$

and it is evident that $g^{\prime}(\mu)>0$, so $g(\mu)$ is an increasing function. Equation (2.34) is the same as

$$
g(\mu)+g(-\mu)+1=0 .
$$

For $\mu>0$ it is easy to see that $g(-\mu)$ is bounded above by

$$
g(-\mu) \leqq \frac{N}{\varepsilon N / n+\mu},
$$

where equality holds if all the $\lambda_{i}^{2}$ are equal. Hence the sum of the positive roots of (2.50) are bounded below by the sum of the positive roots of

$$
g(\mu)+\frac{N}{\varepsilon N / n+\mu}+1=0 .
$$

Equation (2.52) has $n$ positive roots which we write $\gamma_{1}, \ldots, \gamma_{n}$, and one negative root which we denote by $-\delta$. By examining the coefficients of $(2.52)$ we have the identity

$$
\sum_{i=1}^{n} \gamma_{i}-\delta=\varepsilon N-\varepsilon N / n
$$

Next we change $\mu$ to $-\mu$ so that $\delta$ becomes the unique positive root of

$$
\frac{N}{\varepsilon N / n-\mu}+g(-\mu)+1=0 \text {. }
$$

Using (2.51) again we conclude that $\delta$ is bounded below by the positive root of

$$
\frac{N}{\varepsilon N / n-\mu}+\frac{N}{\varepsilon N / n+\mu}+1=0 \text {. }
$$


It is easy to calculate the roots of (2.55), and so we obtain

$$
\delta \geqq N n^{-1}\left(\varepsilon^{2}+2 \varepsilon n\right)^{1 / 2} .
$$

Thus from (2.53) we have

$$
\begin{aligned}
\sum_{i=1}^{n} \beta_{i}-\sum_{i=1}^{n} \lambda_{i}^{2}(1+\varepsilon) & \geqq \sum_{i=1}^{n} \gamma_{i}-N(1+\varepsilon)=\delta-\varepsilon N / n-N \\
& \geqq N\left[n^{-1}\left(\varepsilon^{2}+2 \varepsilon n\right)^{1 / 2}-\varepsilon / n-1\right],
\end{aligned}
$$

which is the inequality we wished to prove.

In the case when all the $\lambda_{i}^{2}$ are equal, $n-1$ of the positive roots of (2.50) are given by $\varepsilon N / n$, while the $n^{\text {th }}$ root is the positive root of (2.55). Thus we get equality in (2.48). Q.E.D.

From (2.20) and (2.48) we have the inequality

$$
J(\psi) \geqq-\varepsilon \sum_{i=1}^{n} \lambda_{i}^{2} N_{i}+N\left[n^{-1}\left(\varepsilon^{2}+2 \varepsilon n\right)^{1 / 2}-\varepsilon / n-1\right],
$$

where $N_{i}$ is the number of particles in the momentum states $a_{i}, b_{i}$, so

$$
N_{i}=\left\langle\psi\left|a_{i}^{*} a_{i}+b_{i}^{*} b_{i}\right| \psi\right\rangle .
$$

Let us suppose that $\varepsilon$ is large, so we may expand the square root in (2.58) as

$$
n^{-1}\left(\varepsilon^{2}+2 \varepsilon n\right)^{1 / 2}=1+\frac{\varepsilon}{n}-\frac{n}{2 \varepsilon}+\ldots
$$

Further let us suppose all the $N_{i}$ are identical, $N_{i}=\kappa, i=1, \ldots, n$, and all the $\lambda_{i}^{2}$ are the same. Thus from (2.58) and (2.60) we have for large $\varepsilon$,

$$
J(\psi) \geqq-\varepsilon N \kappa-N n / 2 \varepsilon .
$$

We can minimize the right side of (2.61) with respect to $\varepsilon$. The minimum occurs for $\varepsilon^{2}=n / 2 \kappa$, which yields

$$
J(\psi) \geqq-\sqrt{2} N(n \kappa)^{1 / 2} .
$$

Hence it seems from (2.62) that we need $\kappa$ of the order of $1 / n$ to achieve the lower bound in (2.47).

We wish next to state our conclusions from the previous calculations as a kind of principle and apply this principle to the Coulomb gas.

Bogoliubov's Principle. Let $N$ and $n$ be fixed. Then, to construct a wave function $\psi$ at least cost of kinetic energy with $J(\psi)$ of order $-N$, one should take all the $\lambda_{i}^{2}$ equal and all the $N_{i}$ of order $1 / n$.

We consider a Coulombic gas in the cube $Q$. Thus we may take $v(k)$ to be

$$
v(k)=1 / L|k|^{2} .
$$

We put most of the Bosons in the states $a_{m}$ with $|m| \leqq \gamma$, with an equal number $\eta$ in each state. Thus we have

$$
N \simeq \gamma^{3} \eta
$$


For $\gamma<|k|<T$, we put $1 / \gamma^{3}$ Bosons in each state $a_{k}$. Hence for such $k$ we have, according to our principle,

$$
J_{k}(\psi) \simeq-N .
$$

Next we compute the energy for such a wave function. The kinetic energy is about

$$
\gamma^{5} \eta / L^{2}+T^{5} / \gamma^{3} L^{2}
$$

For potential energy note that with $|k|<T$ we have the approximation (2.65), while for $|k|>T$ we may take $J_{k}(\psi) \simeq 0$. Hence potential energy is about

Thus we have

$$
-N \sum_{|k|<T} \frac{1}{L|k|^{2}} \simeq-\frac{T}{L} N .
$$

$$
\frac{N^{\alpha}}{\varrho^{1 / 3}}\left\langle\psi, K_{N} \psi\right\rangle+\mathscr{E}\left(|\psi|^{2}\right) \simeq\left\{N^{\alpha}\left[N^{1 / 3} \gamma^{2}+T^{5} / \gamma^{3} N^{2 / 3}\right]-T N^{2 / 3}\right\} \varrho^{1 / 3} .
$$

If for fixed $T$ we minimize the right side of (2.68) with respect to $\gamma$ we obtain

$$
\left\{C N^{\alpha-1 / 15} T^{2}-T N^{2 / 3}\right\} \varrho^{1 / 3},
$$

where $C$ is a universal constant. On minimizing (2.69) for $T>0$ we get

for some constant $C>0$.

$$
-C N^{7 / 5-\alpha} \varrho^{1 / 3},
$$

In the next two sections we shall be concerned with making the above heuristics rigorous and so prove Theorem 1.4. There are two things to be done. First we must approximate the Coulomb potential by a potential which is periodic on $Q$. Second we need to deal with the quartic nature of $J_{k}(\psi)$. In Bogoliubov's method $J_{k}(\psi)$ was reduced to an expression quadratic in the operators $a_{k}$. In the rigorous theory we shall make the kinetic energy quartic by using the fact that $\psi$ is an eigenfunction of the number operator.

\section{Approximating the Coulomb Potential by a Periodic Potential}

Our first purpose here is to show that we may restrict the range of $\alpha$ to the region $2 / 5 \leqq \alpha \leqq 2 / 3$. To show this we need two lemmas, the first of which is well known.

Lemma 3.1. Let $\psi, K_{N}, \Lambda$ be as in Theorem 1.4. Then there is a universal constant $C$ such that

$$
\left\langle\psi, K_{N} \psi\right\rangle \geqq C N^{1 / 3} \varrho^{2 / 3} .
$$

Proof. On using the inequality of Hoffman-Ostenhoff [7] and the uncertainty principle [11] we have

$$
\begin{aligned}
\left\langle\psi, K_{N} \psi\right\rangle & \geqq \int_{A}[\nabla \sqrt{\varrho}(x)]^{2} d x \geqq \frac{1}{4} \int_{A} \frac{\varrho(x)}{|x|^{2}} d x \\
& \geqq C(\operatorname{vol} \Lambda)^{-2 / 3} \int_{\Lambda} \varrho(x) d x=C N^{1 / 3} \varrho^{2 / 3} .
\end{aligned}
$$


Lemma 3.1 gives a lower bound on the kinetic energy. Our next lemma gives a lower bound on the exchange energy.

Lemma 3.2. Let $\psi, A, b$, be as in Theorem 1.4. Then there is a constant $C(b)$ depending only on $b$ such that

$$
\mathscr{E}\left(|\psi|^{2}\right) \geqq-C(b)\left[N \varrho^{1 / 3}+\int_{\Lambda} \sqrt{\varrho}(-\Delta)^{1 / 2} \sqrt{\varrho}(x) d x\right] .
$$

Proof. Let $\mathscr{E}_{\phi}$ denote the exchange energy corresponding to the potential $\phi(x)$. Then it is known [2] that

$$
\mathscr{E}_{\phi}\left(|\psi|^{2}\right) \geqq-\frac{1}{2} N \phi(0),
$$

provided $\phi(x)$ is a positive definite function. We write the Coulomb potential $1 / r$ as

$$
1 / r=\int_{0}^{\infty} e^{-u r} d u
$$

and take $\phi(x)$ as

$$
\phi(x)=\int_{0}^{e^{1 / 3}} e^{-u r} d u
$$

whence we have from (3.4) that

$$
\mathscr{E}_{\phi}\left(|\psi|^{2}\right) \geqq-\frac{1}{2} N \varrho^{1 / 3} .
$$

It follows then that the Coulomb exchange energy $\mathscr{E}\left(|\psi|^{2}\right)$ satisfies the inequality

$$
\mathscr{E}\left(|\psi|^{2}\right) \geqq-\frac{1}{2} N \varrho^{1 / 3}-\frac{1}{2} \iint \frac{\varrho(x) \varrho(y)}{|x-y|} e^{-\varrho^{1 / 3}|x-y|} d x d y .
$$

We need to bound the second term on the right in (3.8) appropriately. First observe that

$$
\iint_{|x-y|<1 / b \varrho^{1 / 3}} \frac{\varrho(x) \varrho(y)}{|x-y|} d x d y \leqq C\left[b N \varrho^{1 / 3}+\int_{\Lambda} \sqrt{\varrho}(-\Delta)^{1 / 2} \sqrt{\varrho}(x) d x\right],
$$

for some universal constant $C$. This follows from the fact that $f(x) \leqq b \varrho^{1 / 3}$ for $x \in \Lambda$ and the Fefferman-Phong technique [5]. The argument is presented in full in [2]. Next we have

$$
\begin{aligned}
& \iint_{|x-y|>1 / b \varrho^{1 / 3}} \frac{\varrho(x) \varrho(y)}{|x-y|} e^{-\varrho^{1 / 3}|x-y|} d x d y \leqq \sum_{r=1}^{\infty} b \varrho^{1 / 3} r^{-1} e^{-r / b} \\
& \cdot \int_{|x-y|<(r+1) / b \varrho^{1 / 3}} \varrho(x) \varrho(y) d x d y .
\end{aligned}
$$

Again using the fact that $f(x) \leqq b \varrho^{1 / 3}$, we see that

$$
\int_{|x-y|<(r+1) / b \varrho^{1 / 3}} \varrho(y) d y \leqq C r^{3},
$$

for some universal constant $C$.

The result (3.3) follows now from (3.8), (3.9), (3.10), and (3.11). Q.E.D. 
Theorem 3.3. Theorem 1.4 holds if $\alpha \leqq 2 / 5$ or $\alpha>2 / 3$.

Proof. We observe that

$$
\begin{aligned}
\frac{N^{\alpha}}{2 \varrho^{1 / 3}}\left\langle\psi, K_{N} \psi\right\rangle \geqq & \frac{N^{\alpha}}{2 \varrho^{1 / 3}} \int_{\Lambda} \sqrt{\varrho}(-\Delta) \sqrt{\varrho}(x) d x \geqq C(b) \int_{\Lambda} \sqrt{\varrho}(-\Delta)^{1 / 2} \sqrt{\varrho}(x) d x \\
& -C(b)^{2} 2^{-1} N^{1-\alpha} \varrho^{1 / 3} .
\end{aligned}
$$

Thus from (3.3) and (3.1) we have that

$\frac{N^{\alpha}}{\varrho^{1 / 3}}\left\langle\psi, K_{N} \psi\right\rangle+\mathscr{E}\left(|\psi|^{2}\right) \geqq C_{1} N^{1 / 3+\alpha} \varrho^{1 / 3}-C_{2}(b) N \varrho^{1 / 3}-C_{3}(b) N^{1-\alpha} \varrho^{1 / 3}$.

It is evident that for $\alpha \leqq 2 / 5$ the above is bounded below by the right side of (1.15). If $\alpha>2 / 3$ then for large $N$ it is positive, in which case it is also bounded below by the right side of (1.15). We have proved the theorem. Q.E.D.

Theorem 3.3 shows us that we need only prove Theorem 1.4 for $\alpha$ in the region $2 / 5 \leqq \alpha \leqq 2 / 3+\eta$ for some $\eta>0$. From now on we shall restrict ourselves to such $\alpha$. There is a particular value of $\alpha$ in this region, namely $\alpha=8 / 15$, which has significance for us. The reason is that this value of $\alpha$ solves the equation

$$
N^{1 / 3+\alpha}=N^{7 / 5-\alpha} \text {. }
$$

Since the kinetic energy term in $(1.15)$ is bounded below by a positive constant times $N^{1 / 3+\alpha} \varrho^{1 / 3}$, we should for $\alpha>8 / 15$ bound the potential energy terms in (1.15) by a negative constant times $N^{1 / 3+\alpha} \varrho^{1 / 3}$, while for $\alpha<8 / 15$ by a negative constant times $N^{7 / 5-\alpha} \varrho^{1 / 3}$.

Equation (3.5) represents the Coulomb potential as an integral. Next we subtract off parts of this integral and bound the corresponding exchange energies in a similar spirit to that used in Theorem 3.3.

Lemma 3.4. For $2 / 5 \leqq \alpha \leqq 8 / 15$, let $\phi_{\alpha}(x)$ be the potential defined $b y$

$$
\phi_{\alpha}(x)=\int_{0}^{\varrho^{1 / 3} N^{2 / 5-\alpha}} e^{-u|x|} d u+\int_{\varrho^{1 / 3} N^{\alpha / 2-1 / 5}}^{\infty} e^{-u|x|} d u .
$$

Then there is a constant $C(b)$ depending only on $b$ such that

$$
\frac{N^{\alpha}}{2 \varrho^{1 / 3}}\left\langle\psi, K_{N} \psi\right\rangle+\mathscr{E}_{\phi_{\alpha}}\left(|\psi|^{2}\right) \geqq-C(b) N^{7 / 5-\alpha} \varrho^{1 / 3} .
$$

Proof. The potential $\phi_{\alpha}(x)$ has been written as a sum of two terms,

$$
\phi_{\alpha}(x)=\Phi_{1}(x)+\Phi_{2}(x)
$$

with $\Phi_{1}(x)$ given by

$$
\Phi_{1}(x)=\int_{0}^{\varrho^{1 / 3}} e^{-u|x|} d u
$$

From (3.4) we see that

$$
\mathscr{E}_{\Phi_{1}}\left(|\psi|^{2}\right) \geqq-\frac{1}{2} N^{7 / 5-\alpha} \varrho^{1 / 3} .
$$

We therefore have only to deal with $\Phi_{2}$. 
It will be sufficient for us to show that

$$
\frac{N^{\alpha}}{2 \varrho^{1 / 3}}\left\langle\psi, K_{N} \psi\right\rangle-\frac{1}{2} \iint \varrho(x) \varrho(y) \Phi_{2}(x-y) d x d y \geqq-C(b) N^{7 / 5-\alpha} \varrho^{1 / 3} .
$$

We proceed similarly to (3.9) and (3.10). Putting $\gamma=N^{\alpha / 2-1 / 5}$, we see as in (3.9) that there is a constant $C(b)$ such that

$$
\begin{aligned}
& \quad \iint_{|x-y|<1 / \gamma \varrho^{1 / 3}} \frac{\varrho(x) \varrho(y)}{|x-y|} d x d y \\
& \leqq C(b)\left[\gamma \varrho^{1 / 3} \iiint_{|x-y|<c / \gamma \varrho^{1 / 3}} \varrho(x) \varrho(y) d x d y+\int_{\Lambda} \sqrt{\varrho}(-\Delta)^{1 / 2} \sqrt{\varrho}(x) d x\right],
\end{aligned}
$$

where $c>1$ is a universal constant. Arguing then as in (3.10) and Theorem 3.3, it is clear that we need only prove that for any $r>1$ and constant $C_{1}(b)$ there is a constant $C_{2}(b)$ such that

$$
\begin{aligned}
& \frac{N^{\alpha}}{\varrho^{1 / 3}} \int_{\Lambda}[\nabla \sqrt{\varrho}(x)]^{2} d x \\
& \quad-C_{1}(b) \gamma \varrho^{1 / 3} / r^{3} \iint_{|x-y|<r / \gamma \varrho^{1 / 3}} \varrho(x) \varrho(y) d x d y \geqq-C_{2}(b) N^{7 / 5-\alpha} \varrho^{1 / 3} .
\end{aligned}
$$

The inequality (3.22) is evident if $r>\gamma / b$, since we know $f(x) \leqq b \varrho^{1 / 3}$ all $x \in \Lambda$. For $r<\gamma / b$ the result follows from the next lemma, and we are done. Q.E.D.

Lemma 3.5. Let $Q$ be a cube in $\mathbb{R}^{3}$, and for some integer $m>0$ suppose $Q$ is divided into $2^{3 m}=n$ dyadic subcubes $Q_{1}, \ldots, Q_{n}$. Let $\psi(x)$ be a function defined for $x \in Q$, and define $\sigma_{i}, i=1, \ldots, n, b y$

$$
\sigma_{i}=\int_{Q_{\imath}} \psi^{2}(x) d x
$$

Then, if $L$ is the length of a side of $Q$, for any $\varepsilon>0$ there is a constant $C(\varepsilon)>0$ such that

$$
\varepsilon L^{2} n^{1 / 3} \int_{Q} \psi^{2}(x) d x \int_{Q}(\nabla \psi)^{2}(x) d x-\sum_{i=1}^{n} \sigma_{i}^{2} \geqq-\frac{C(\varepsilon)}{n}\left[\int_{Q} \psi^{2}(x) d x\right]^{2} .
$$

Proof. We first obtain a lower bound on the gradient term in terms of the $\sigma_{i}$, $i=1, \ldots, n$. To do this let $(x, y, z)$ be coordinates in $\mathbb{R}^{3}$ and suppose that $Q_{1}$ and $Q_{2}$ are adjacent with a common face in the $(y, z)$ plane. Then we have

$$
\begin{aligned}
\mid \sigma_{1} & -\sigma_{2} \mid \\
= & \mid \int_{0}^{L / n^{1 / 3}} \int_{0}^{L / n^{1 / 3}} \int_{0}^{L / n^{1 / 3}}\left[\psi^{2}\left(x+L / n^{1 / 3}, y, z\right)-\psi^{2}(x, y, z) d x d y d z \mid\right. \\
\leqq & \left|\psi\left(x+L / n^{1 / 3}, y, z\right)-\psi(x, y, z)\right|\left|\psi\left(x+L / n^{1 / 3}, y, z\right)+\psi(x, y, z)\right| d x d y d z \\
\leqq & {\left[\int\left|\psi\left(x+L / n^{1 / 3}, y, z\right)-\psi(x, y, z)\right|^{2} d x d y d z\right]^{1 / 2} } \\
& \cdot\left[\int\left|\psi\left(x+L / n^{1 / 3}, y, z\right)+\psi(x, y, z)\right|^{2} d x d y d z\right]^{1 / 2} .
\end{aligned}
$$


The second integral in the last expression is evidently bounded above by $2\left(\sigma_{1}+\sigma_{2}\right)$. The first integral is equal to

$$
\begin{gathered}
\iiint d y d z d x\left[\int_{x}^{x+L / n^{1 / 3}} \frac{\partial \psi}{\partial t}(t, y, z) d t\right]^{2} \leqq \iiint d y d z d x \frac{L}{n^{1 / 3}} \int_{x}^{x+L / n^{1 / 3}}\left[\frac{\partial \psi}{\partial t}\right]^{2} d t \\
\leqq \frac{L^{2}}{n^{2 / 3}} \int_{Q_{1} \cup Q_{2}}(\nabla \psi)^{2}(x) d x .
\end{gathered}
$$

We conclude therefore that

$$
L^{2} n^{1 / 3} \int_{Q_{1} \cup Q_{2}}(\nabla \psi)^{2}(x) d x \geqq n \frac{\left(\sigma_{1}-\sigma_{2}\right)^{2}}{2\left(\sigma_{1}+\sigma_{2}\right)} .
$$

From (3.27) and (3.24) we see that it is sufficient for us to prove that

$$
\varepsilon n\left[\sum_{i=1}^{n} \sigma_{i}\right]_{i=1}^{n-1} \frac{\left(\sigma_{i}-\sigma_{i+1}\right)^{2}}{2\left(\sigma_{i}+\sigma_{i+1}\right)}-\sum_{i=1}^{n} \sigma_{i}^{2} \geqq-\frac{C(\varepsilon)}{n}\left[\sum_{i=1}^{n} \sigma_{i}\right]^{2} .
$$

To do this note that for $t<r$ we have

$$
\begin{aligned}
\left|\sigma_{t}-\sigma_{r}\right| & =\left|\sum_{i=t}^{r-1}\left(\sigma_{i}-\sigma_{i+1}\right)\right| \leqq \sum_{i=1}^{n-1} \frac{\left|\sigma_{i}-\sigma_{i+1}\right|}{\left[2\left(\sigma_{i}+\sigma_{i+1}\right)\right]^{1 / 2}}\left[2\left(\sigma_{i}+\sigma_{i+1}\right)\right]^{1 / 2} \\
& \leqq 2\left[\sum_{i=1}^{n-1} \frac{\left(\sigma_{i}-\sigma_{i+1}\right)^{2}}{2\left(\sigma_{i}+\sigma_{i+1}\right)}\right]^{1 / 2}\left[\sum_{i=1}^{n} \sigma_{i}\right]^{1 / 2} .
\end{aligned}
$$

Since $t$ and $r$ are arbitrary it follows from (3.29) that the left side of (3.28) is bounded below by

$$
\varepsilon n / 4\left(\max \sigma_{i}-\min \sigma_{i}\right)^{2}-\sum_{i=1}^{n} \sigma_{i}^{2} .
$$

It is now clear that (3.30) is bounded below by the right side of (3.28) Q.E.D.

Next we deal with the case where $\alpha>8 / 15$. We have the following lemma:

Lemma 3.6. For $\alpha>8 / 15$, let $\phi_{\alpha}(x)$ be the potential defined by

$$
\phi_{\alpha}(x)=\int_{0}^{c \varrho^{1 / 3} N^{\alpha-2 / 3}} e^{-u|x|} d u+\int_{c(b) \varrho^{1 / 3} N^{1 / 3-\alpha / 2}}^{\infty} e^{-u|x|} d u,
$$

where $c$ is a universal constant and $c(b)$ is a constant depending only on $b$. The constants $c$ and $c(b)$ may be chosen to be positive numbers such that for all $\psi$ and $\alpha$,

$$
\frac{N^{\alpha}}{2 \varrho^{1 / 3}}\left\langle\psi, K_{N} \psi\right\rangle+\mathscr{E}_{\phi_{\alpha}}\left(|\psi|^{2}\right) \geqq 0 .
$$

Proof. We argue similarly to Lemma 3.4. The potential $\phi_{\alpha}(x)$ has been written as a sum

$$
\phi_{\alpha}(x)=\Phi_{1}(x)+\Phi_{2}(x)
$$

with $\Phi_{1}(x)$ given by

$$
\Phi_{1}(x)=\int_{0}^{c Q^{1 / 3} N^{\alpha-2 / 3}} e^{-u|x|} d u
$$


From (3.4) we have that

$$
\mathscr{E}_{\Phi_{1}}\left(|\psi|^{2}\right) \geqq-\frac{1}{2} c N^{1 / 3+\alpha} \varrho^{1 / 3} .
$$

From Lemma 3.1 it is clear we may choose the constant $c$ in (3.35) such that

$$
\frac{N^{\alpha}}{4 \varrho^{1 / 3}}\left\langle\psi, K_{N} \psi\right\rangle+\mathscr{E}_{\Phi_{1}}\left(|\psi|^{2}\right) \geqq 0 .
$$

To deal with $\Phi_{2}$ we argue exactly as in Lemma 3.4, proving inequalities similar to (3.21), (3.22) and making use of Lemma 3.5. We conclude that for $c(b)$ sufficiently large, depending only on $b$, we have

$$
\frac{N^{\alpha}}{4 \varrho^{1 / 3}}\left\langle\psi, K_{N} \psi\right\rangle+\mathscr{E}_{\Phi_{2}}\left(|\psi|^{2}\right) \geqq 0 .
$$

This completes the proof. Q.E.D.

Remark. Note that Lemmas 3.4 and 3.6 are very easy if the one point function $\varrho(x)$ is identically constant in $\Lambda$. The condition $f(x) \leqq b \varrho^{1 / 3}$ in $\Lambda$ means that $\varrho(x)$ is on average constant in $\Lambda$. The purpose of Lemma 3.5 is to show that because of the kinetic energy the estimates for $\varrho(x)$ constant continue to hold when $\varrho(x)$ is allowed to vary slightly.

We have shown so far that if we replace the Coulomb potential by the potential $\phi_{\alpha}(x)$ then the conclusion of Theorem 1.4 holds. This leaves us to deal with the potential $1 /|x|-\phi_{\alpha}(x)$. We claim that this potential may be well approximated by a periodic potential. One can see easily why this should be so. For $\alpha \leqq 8 / 15$ it is an integral in $e^{-u|x|}$ with $u \geqq \varrho^{1 / 3} N^{2 / 5-\alpha}$. We may assume without loss of generality that $\Lambda$ is a box with center at the origin. As $x$ varies in $\Lambda,|x|$ varies from 0 to $(\operatorname{Vol} \Lambda)^{1 / 3}=N^{1 / 3} \varrho^{-1 / 3}$. Thus for $u \geqq \varrho^{1 / 3} N^{2 / 5-\alpha}, u|x|$ varies from 0 to something larger than $N^{11 / 15-\alpha}$, which goes to $\infty$ with $N$. Hence we expect to be able to approximate $e^{-u|x|}$ by something periodic. A similar argument applies in the case when $\alpha>8 / 15$.

To implement this scheme let $Q_{\Lambda}$ be a cube concentric with $\Lambda$ but with side which has 4 times the length of a side of $\Lambda$. It is clear that if $x, y \in \Lambda$ then $x-y \in Q_{\Lambda}$. Since the wave function $\psi$ in Theorem 1.4 is supported in $\Lambda^{N}$, the potential enters in $(1.15)$ with argument $x-y$ where $x, y \in \Lambda$. Hence we may expand $1 /|x|-\phi_{\alpha}(x)$ in a Fourier series on $Q_{A}$ and this will give a good representation for the potential in the situation of Theorem 1.4. The problem is that this new periodic potential is no longer positive definite. However, because of the considerations of the previous paragraph, the Fourier coefficients $v(k)$ can only be negative for very large values of $k$ depending on $N$.

In the following lemma we shall make use of the fact that for $\lambda>0$,

$$
\begin{aligned}
\int_{|x|<\lambda} e^{-|x|} e^{i x \cdot \xi} d x & \\
= & \frac{8 \pi}{\left(1+|\xi|^{2}\right)^{2}} \frac{-4 \pi \lambda e^{-\lambda}}{\left(1+|\xi|^{2}\right)}\left[\frac{\sin \lambda|\xi|}{|\xi|}+\cos \lambda|\xi|\right] \\
& \quad \frac{+4 \pi e^{-\lambda}}{\left(1+|\xi|^{2}\right)^{2}}\left[\left(|\xi|^{2}-1\right) \frac{\sin \lambda|\xi|}{|\xi|}-2 \cos \lambda|\xi|\right] .
\end{aligned}
$$


Let us define $I_{\lambda}(\xi)$ by

$$
I_{\lambda}(\xi)=\int_{|x|<\lambda} e^{-|x|} e^{-i x \cdot \xi} d x .
$$

From (3.38) it is easy to see that we may find universal constants $C_{1}$ and $C_{2}$ such

$$
\begin{gathered}
0 \leqq I_{\lambda}(\xi) \leqq \frac{16 \pi}{\left(1+|\xi|^{2}\right)^{2}} \text { if }|\xi| \leqq C_{1} e^{\lambda / 3} \\
\left|I_{\lambda}(\xi)\right| \leqq \frac{8 \pi}{\left(1+|\xi|^{2}\right)^{2}}+\frac{C_{2} e^{-\lambda / 2}}{1+|\xi|^{2}}, \quad \xi \in \mathbb{R}^{3} .
\end{gathered}
$$

Lemma 3.7. Let $Q_{A}$ have side of length $L$. Then for $x \in Q_{\Lambda}$ with $|x|<L / 2$ the potential $1 /|x|-\phi_{\alpha}(x)$ may be expanded in a Fourier series

$$
1 /|x|-\phi_{\alpha}(x)=\sum_{k \in Z^{3}} v_{\alpha}(k) e^{2 \pi i k \cdot x / L} .
$$

There is a universal constant $C$ such that for $|k|$ satisfying

$$
|k| \leqq C \exp \left[N^{1 / 6}\right] \text {, }
$$

then the $v_{\alpha}(k)$ obey the inequalities

$$
\begin{gathered}
0 \leqq v_{\alpha}(k) \leqq \frac{C^{\prime} N^{\alpha-1 / 15} \varrho^{1 / 3}}{\left(k^{2}+1\right)\left(k^{2}+N^{\alpha+4 / 15}\right)} \text { if } \alpha \leqq 8 / 15, \\
0 \leqq v_{\alpha}(k) \leqq \frac{C^{\prime}(b) N^{1-\alpha} \varrho^{1 / 3}}{\left(k^{2}+1\right)\left(k^{2}+C(b) N^{4 / 3-\alpha}\right)}
\end{gathered}
$$

if $\alpha>8 / 15$, where $C$ is a universal constant and $C(b), C^{\prime}(b)$ depend only on $b$.

If $k$ does not satisfy (3.42) then there are constants $C^{\prime \prime}$ and $C^{\prime \prime}(b)$ such that

$$
\begin{gathered}
\left|v_{\alpha}(k)\right| \leqq C^{\prime \prime} \exp \left[-N^{1 / 6}\right] \varrho^{1 / 3} /|k|^{2}, \quad \text { if } \alpha \leqq 8 / 15, \\
\left|v_{\alpha}(k)\right| \leqq C^{\prime \prime}(b) \exp \left[-N^{1 / 6}\right] \varrho^{1 / 3} /|k|^{2}, \quad \text { if } \quad \alpha>8 / 15 .
\end{gathered}
$$

Proof. We shall deal only with the case $\alpha \leqq 8 / 15$, since $\alpha>8 / 15$ is similar. Then it is evident from (3.15) that we may take $v_{\alpha}(k)$ to be

$$
v_{\alpha}(k)=\int_{e^{1 / 3} N^{2 / 5-\alpha}}^{\varrho^{1 / 3} N^{\alpha / 2-1 / 5}} \frac{1}{L^{3} u^{3}} I_{L u / 2}\left(\frac{2 \pi k}{L u}\right) d u .
$$

Now it is clear that there is a universal constant $C$ such that if

$$
|k| \leqq C \exp \left[N^{1 / 6}\right]
$$

then we have for all $u \geqq Q^{1 / 3} N^{2 / 5-\alpha}$,

$$
\left|\frac{2 \pi k}{L u}\right| \leqq C_{1} e^{u L / 6}
$$

Thus for $k$ satisfying (3.48) we have from (3.40) the inequality

$$
0 \leqq v_{\alpha}(k) \leqq \int_{\varrho^{1 / 3} N^{2 / 5-\alpha}}^{\varrho^{1 / 3} N^{\alpha / 2-1 / 5}} \frac{16 \pi u d u}{L^{3}\left[u^{2}+(2 \pi k / L)^{2}\right]^{2}} .
$$


It is evident that (3.50) implies there is a universal constant $C^{\prime}$ such that for $k$ satisfying (3.48) we have

$$
0 \leqq v_{\alpha}(k) \leqq \frac{C^{\prime} N^{\alpha-1 / 15} \varrho^{1 / 3}}{\left(k^{2}+1\right)\left(k^{2}+N^{\alpha+4 / 15}\right)} .
$$

Next we consider $k$ which do not satisfy (3.48). If we use the second inequality in (3.40) it is easy to see that for such $k$ there is a universal constant $C^{\prime \prime}$ such that

$$
\left|v_{\alpha}(k)\right| \leqq C^{\prime \prime} \exp \left[-N^{1 / 6}\right] \varrho^{1 / 3} /|k|^{2} .
$$

Since (3.51) is the same as (3.43) and (3.52) as (3.45) we are finished. Q.E.D.

Lemma 3.7 divides the $v_{\alpha}(k)$ into two kinds depending on the value of $k$. We define potentials $V_{\alpha}(x)$ and $U_{\alpha}(x)$ by

$$
\begin{gathered}
V_{\alpha}(x)=\sum_{|k| \leqq C \exp \left[N^{1 / 6}\right]} v_{\alpha}(k) e^{2 \pi i k \cdot x / L}, \\
U_{\alpha}(x)=1 /|x|-\phi_{\alpha}(x)-V_{\alpha}(x) .
\end{gathered}
$$

Observe that all the Fourier coefficients of $V_{\alpha}(x)$ are non-negative. The potential $U_{\alpha}(x)$ is a remainder term which we shall now bound.

Lemma 3.8. Let $\psi$ be supported in $\Lambda^{N}$. Then for all $N$ sufficiently large,

$$
\frac{N^{\alpha}}{4 \varrho^{1 / 3}}\left\langle\psi, K_{N} \psi\right\rangle+\mathscr{E}_{U_{\alpha}}\left(|\psi|^{2}\right) \geqq 0 .
$$

Proof. For $x, y \in \Lambda, x-y$ satisfies the inequality $|x-y|<L / 2$. Hence in our situation $U_{\alpha}$ is well represented by the Fourier series,

$$
U_{\alpha}(x)=\sum_{|k|>C \exp \left[N^{1 / 6}\right]} v_{\alpha}(k) e^{2 \pi i k \cdot x / L} .
$$

Also since $\Lambda \subset Q_{\Lambda}$, we may take $\psi$ to be periodic on $Q_{\Lambda}$. We are therefore justified in writing the expression (3.55) in second quantised form as was already done in Sect. 2.

The expression in (3.55) is given in second quantised form as

$$
\frac{N^{\alpha}}{4 \varrho^{1 / 3}}\left\langle\psi\left|\frac{4 \pi^{2}}{L^{2}} \sum_{k \in Z^{3}} k^{2} a_{k}^{*} a_{k}\right| \psi\right\rangle+\frac{1}{2} \sum_{|k|>C \exp \left[N^{1 / 6}\right]} v_{\alpha}(k) J_{k}(\psi),
$$

with $J_{k}(\psi)$ defined in (2.10). Now we have

$$
\left|\left\langle\psi\left|A_{k}\right| \psi\right\rangle\right| \leqq \sum_{n}\left\langle\psi\left|a_{n+k}^{*} a_{n+k}\right| \psi\right\rangle^{1 / 2}\left\langle\psi\left|a_{n}^{*} a_{n}\right| \psi\right\rangle^{1 / 2},
$$

by the Schwarz inequality. On the other hand from (2.5) we have

$$
\begin{aligned}
& \left.\left|J_{k}(\psi)+\right|\left\langle\psi\left|A_{k}\right| \psi\right\rangle\right|^{2}|=|\left\langle\psi\left|\sum_{n, r} a_{n+k}^{*} a_{r-k}^{*} a_{n} a_{r}\right| \psi\right\rangle \mid \\
& \quad \leqq \sum_{n, r}\left\langle\psi\left|a_{n+k}^{*} a_{r-k}^{*} a_{r-k} a_{n+k}\right| \psi\right\rangle^{1 / 2} \cdot\left\langle\psi\left|a_{n}^{*} a_{r}^{*} a_{r} a_{n}\right| \psi\right\rangle^{1 / 2} \\
& \quad \leqq \sum_{n}\left\langle\psi\left|a_{n+k}^{*} n a_{n+k}\right| \psi\right\rangle^{1 / 2} \cdot\left\langle\psi\left|a_{n}^{*} n a_{n}\right| \psi\right\rangle^{1 / 2},
\end{aligned}
$$


where $n$ is the number operator (2.4). If we commute $n$ with the operators $a_{n+k}$ and $a_{n}$ in the above inequality and use the fact that $\psi$ is an eigenfunction of $n$ with eigenvalue $N$, we see that the last expression is bounded above by

$$
\sum_{n}(N-1)\left\langle\psi\left|a_{n+k}^{*} a_{n+k}\right| \psi\right\rangle^{1 / 2}\left\langle\psi\left|a_{n}^{*} a_{n}\right| \psi\right\rangle^{1 / 2} .
$$

If we put $N_{m}=\left\langle\psi\left|a_{m}^{*} a_{m}\right| \psi\right\rangle$ for $m \in Z^{3}$, then from (3.58) and (3.60) we have

$$
\left|J_{k}(\psi)\right| \leqq 2 N \sum_{m} N_{m+k}^{1 / 2} N_{m}^{1 / 2} .
$$

Next we turn to the kinetic energy. We write

$$
\begin{aligned}
& \sum_{k} k^{2} N_{k}=\frac{1}{N} \sum_{k, m} k^{2} N_{k} N_{m}=\frac{1}{N} \sum_{k, m}(m+k)^{2} N_{m+k} N_{m} \\
& \geqq \frac{1}{4 N} \sum_{|m| \leqq|k| / 2} k^{2} N_{m+k} N_{m} \\
& \geqq \frac{1}{8 N} \sum_{\{m:|m| \leqq|k| / 2 \text { or }|m+k| \leqq|k| / 2\}} k^{2} N_{m+k} N_{m} \text {, }
\end{aligned}
$$

the last inequality being obtained by adding the $k$ and $-k$ terms.

Next, if we use the elementary inequality

$$
x^{2}-2 a x \geqq-a^{2},
$$

we have from (3.62), (3.52), for any $\varepsilon>0$,

$$
\begin{aligned}
& \frac{N^{\alpha}}{8 \varrho^{1 / 3}}\left\langle\psi\left|K_{N}\right| \psi\right\rangle-\left.\frac{1}{2} N \sum_{\left\{m:|m| \leqq|k|^{1-\varepsilon / 2 \operatorname{or}|m+k|}|k|>C \exp \left[N^{1 / 6}\right]\right.} \sum\right|^{1-\varepsilon / 2\}} \\
& \cdot\left|v_{\alpha}(k)\right| N_{m+k}^{1 / 2} N_{m}^{1 / 2} \geqq-\varrho^{1 / 3} C(\varepsilon) \exp \left[-3 \varepsilon N^{1 / 6}\right],
\end{aligned}
$$

where $C(\varepsilon)$ is a constant depending only on $\varepsilon$.

Next let us define $N(u)$ by

$$
N(u)=\sum_{|m| \geqq u} N_{m}, \quad u>0 .
$$

Then we have

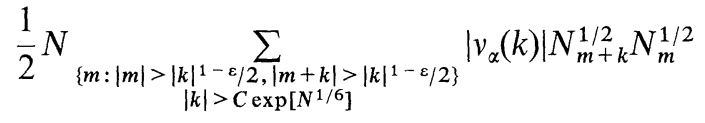

$$
\begin{aligned}
& \leqq \frac{1}{2} N \sum_{|k|>C \exp \left[N^{1 / 6}\right]}^{-}\left|v_{\alpha}(k)\right| N\left(|k|^{1-\varepsilon} / 2\right) \\
& \leqq A \varrho^{1 / 3} \sum_{|k|>C \exp \left[N^{1 / 6}\right]}|k|^{-2} N\left(|k|^{1-\varepsilon} / 2\right) \\
& \leqq B \varrho^{1 / 3} \int_{C \exp \left[N^{1 / 6}\right]}^{\infty} N\left(u^{1-\varepsilon} / 2\right) d u,
\end{aligned}
$$

where $A$ and $B$ are constants. 
We may bound kinetic energy below by an integral in $N(u)$ as follows:

$$
\begin{aligned}
& \frac{N^{\alpha}}{16 \varrho^{1 / 3}}\left\langle\psi, K_{N} \psi\right\rangle \\
& \geqq A^{\prime} \varrho^{1 / 3} \sum_{k} k^{2} N_{k} \geqq A^{\prime} \varrho^{1 / 3} \sum_{j=0}^{\infty} j^{2} \sum_{j \leqq|k|<j+1} N_{k} \\
& \quad=A^{\prime} \varrho^{1 / 3} \sum_{j=0}^{\infty} j^{2}[N(j)-N(j+1)] \\
& \quad=A^{\prime} \varrho^{1 / 3}\left[\sum_{j=0}^{\infty}\left\{j^{2} N(j)-(j+1)^{2} N(j+1)\right\}+\sum_{j=0}^{\infty}(2 j+1) N(j+1)\right] \\
& \quad=A^{\prime} \varrho^{1 / 3} \sum_{j=0}^{\infty}(2 j+1) N(j+1) \geqq A^{\prime} \varrho^{1 / 3} \int_{2}^{\infty} u N(u) d u,
\end{aligned}
$$

where $A^{\prime}$ is a constant times a power of $N$.

The inequality (3.55) follows from (3.61), (3.64), (3.66), (3.67) and Lemma 3.1. Q.E.D.

It is clear now that it is sufficient for us to prove Theorem 1.4 with the Coulomb potential replaced by the periodic potential $V_{\alpha}(x)$. In the next section we shall achieve this by making use of Bogoliubov's method.

\section{Rigorous Version of Bogoliubov's Method}

We shall first assume that $\alpha \leqq 8 / 15$. At the end of Sect. 2 , in the discussion of the Coulomb gas, it was stated that one should put most Bosons in states $m \in Z^{3}$ with $|m| \leqq \gamma$. Here we wish to fix an appropriate value for $\gamma$.

To do this we define operators $S_{k, m}$ and $T_{k, m}$ for $|m| \leqq \gamma$ by

$$
\begin{gathered}
S_{k, m}=a_{m}^{*} a_{m+k}, \quad|m| \leqq \gamma, \\
T_{k, m}=a_{m}^{*} a_{m-k}, \quad 0<|m| \leqq \gamma, \\
T_{k, 0}=a_{0}^{*} a_{-k}+\sum_{\{n:|n|>\gamma,|n+k|>\gamma\}} a_{n+k}^{*} a_{n} .
\end{gathered}
$$

It is evident then from (2.6) that

$$
A_{k}=\sum_{|m| \leqq \gamma}\left[S_{k, m}^{*}+T_{k, m}\right] .
$$

From (2.10) we see on using the commutation relations that $J_{k}(\psi)$ is given by the formula

$$
\begin{aligned}
J_{k}(\psi)= & \left\langle\psi\left|S_{k}^{*} S_{k}+T_{k}^{*} T_{k}+S_{k} T_{k}+S_{k}^{*} T_{k}^{*}\right| \psi\right\rangle-\left|\left\langle\psi\left|S_{k}^{*}+T_{k}\right| \psi\right\rangle\right|^{2} \\
& +\left\langle\psi\left|\sum_{|m| \leqq \gamma}\left[a_{m}^{*} a_{m}-a_{m+k}^{*} a_{m+k}\right]\right| \psi\right\rangle-N,
\end{aligned}
$$


where $S_{k}$ and $T_{k}$ are given by

$$
\begin{aligned}
& S_{k}=\sum_{|m| \leqq \gamma} S_{k, m}, \\
& T_{k}=\sum_{|m| \leqq \gamma} T_{k, m} .
\end{aligned}
$$

Notice that (4.3) looks the same as (2.19) except for the last two terms. It is easy to bound these terms, and we have if $|k| \geqq 2 \gamma$, then

$$
\sum_{|m| \leqq \gamma}\left[N_{m}-N_{m+k}\right]-N \geqq-2 N(\gamma),
$$

where $N(u)$ is defined as in (3.65).

We choose $\gamma$ such that

$$
\begin{aligned}
& N(\gamma) \sum_{k \in Z^{3}} v_{\alpha}(k) \leqq C N^{7 / 5-\alpha} \varrho^{1 / 3}, \\
& N \sum_{|k| \leqq 2 \gamma} v_{\alpha}(k) \leqq C N^{7 / 5-\alpha} \varrho^{1 / 3},
\end{aligned}
$$

for some constant $C$. From (4.6) and (4.7) it follow that we may drop the last 2 terms in (4.3) from our future consideration. From (4.8) and (2.11) we may restrict ourselves to values of $k$ with $|k|>2 \gamma$.

From (3.43) we see that

$$
\begin{gathered}
\sum_{k} v_{\alpha}(k) \leqq C N^{\alpha / 2-1 / 5} \varrho^{1 / 3}, \\
N \sum_{|k| \leqq 2 \gamma} v_{\alpha}(k) \leqq C \gamma N^{2 / 3} \varrho^{1 / 3},
\end{gathered}
$$

where $C$ is a universal constant. Now we may assume that the wave function $\psi$ satisfies

$$
\frac{N^{\alpha}}{\varrho^{1 / 3}}\left\langle\psi, K_{N} \psi\right\rangle+\mathscr{E}\left(|\psi|^{2}\right) \leqq 0 .
$$

Otherwise there is nothing to prove in Theorem 1.4. From (3.3) and (4.11) it follows that for some constant $A$ we have

$$
\frac{N^{\alpha}}{\varrho^{1 / 3}}\left\langle\psi, K_{N} \psi\right\rangle-A N \varrho^{1 / 3} \leqq 0 .
$$

It is now easy to see from the representation (2.3) for $K_{N}$ that $N(\gamma)$ satisfies an inequality

$$
N(\gamma) \leqq A^{\prime} N^{5 / 3-\alpha} \gamma^{-2},
$$

for some universal constant $A^{\prime}$. From (4.9), (4.10), and (4.13) it is evident that for $\alpha$ satisfying $\alpha \leqq 14 / 25$ we may choose $\gamma$ such that (4.7) and (4.8) hold. In fact we may take $\gamma$ to be given by

$$
\gamma=N^{\alpha / 4+1 / 30}
$$


Our next aim is to write the kinetic energy in such a way that it is in a form suitable for applying Bogoliubov transformations. To do this we observe that

$$
\begin{aligned}
\left\langle\psi\left|K_{N}\right| \psi\right\rangle= & \frac{4 \pi^{2}}{L^{2}}\left\langle\psi\left|\sum_{k} k^{2} a_{k}^{*} a_{k}\right| \psi\right\rangle=\frac{4 \pi^{2}}{N L^{2}}\left\langle\psi\left|n \sum k^{2} a_{k}^{*} a_{k}\right| \psi\right\rangle \\
\leqq & \frac{4 \pi^{2}}{N L^{2}} \sum_{|k|>2 \gamma} \frac{1}{2}\langle\psi| \sum_{|m| \leqq \gamma} a_{m}^{*} a_{m}(m+k)^{2} a_{m+k}^{*} a_{m+k} \\
& +\sum_{0<|n| \leqq \gamma} a_{n}^{*} a_{n}(n-k)^{2} a_{n-k}^{*} a_{n-k}|\psi\rangle \\
\geqq & \frac{\pi^{2}}{2 N L^{2}} \sum_{|k|>2 \gamma} k^{2}\langle\psi| \sum_{|m| \leqq \gamma} a_{m}^{*} a_{m} a_{m+k}^{*} a_{m+k} \\
& +\sum_{0<|n| \leqq \gamma} a_{n}^{*} a_{n} a_{n-k}^{*} a_{n-k}|\psi\rangle \\
= & \frac{\pi^{2}}{2 N L^{2}} \sum_{|k|>2 \gamma} k^{2}\langle\psi| \sum_{|m| \leqq \gamma}\left[S_{k, m}^{*} S_{k, m}-a_{m+k}^{*} a_{m+k}\right] \\
& +\sum_{0<|n| \leqq \gamma}\left[T_{k, n}^{*} T_{k, n}-a_{n-k}^{*} a_{n-k}\right]|\psi\rangle \\
\geqq & \frac{\pi^{2}}{2 N L^{2}} \sum_{|k|>2 \gamma} k^{2}\langle\psi| \sum_{|m| \leqq \gamma} S_{k, m}^{*} S_{k, m} \\
& +\sum_{0<|n| \leqq \gamma} T_{k, n}^{*} T_{k, n}|\psi\rangle-\frac{C \gamma^{3}}{N}\left\langle\psi\left|K_{N}\right| \psi\right\rangle,
\end{aligned}
$$

for some universal constant $C$. From (4.14) we see that if $\alpha \leqq 8 / 15$, then $\gamma^{3} \ll N$ for large $N$, and so we conclude that if $N$ is large then

$$
\begin{aligned}
\left\langle\psi, K_{N} \psi\right\rangle \geqq & \frac{\pi^{2}}{4 N L^{2}} \sum_{|k|>2 \gamma} k^{2}\langle\psi| \sum_{|m| \leqq \gamma} S_{k, m}^{*} S_{k, m} \\
& +\sum_{0<|n| \leqq \gamma} T_{k, n}^{*} T_{k, n}|\psi\rangle .
\end{aligned}
$$

Now for $\varepsilon>0$ let us put

$$
\begin{aligned}
I_{k}(\varepsilon)= & 2 \varepsilon\left\langle\psi\left|\sum_{|m| \leqq \gamma} S_{k, m}^{*} S_{k, m}+\sum_{0<|n| \leqq \gamma} T_{k, n}^{*} T_{k, n}\right| \psi\right\rangle \\
& +\left\langle\psi\left|S_{k}^{*} S_{k}+T_{k}^{*} T_{k}+S_{k} T_{k}+S_{k}^{*} T_{k}^{*}\right| \psi\right\rangle-\left|\left\langle\psi\left|S_{k}^{*}+T_{k}\right| \psi\right\rangle\right|^{2} .
\end{aligned}
$$

Thus $I_{k}(\varepsilon)$ is just like the expression (2.20).

Lemma 4.1. Suppose $|k|>2 \gamma$ and $N_{0} \leqq N_{k}$ for all $k$ with $|k| \leqq \gamma$. Let $\beta_{m},|m| \leqq \gamma$ be the absolute values of the negative roots of the polynomial equation

$$
\begin{aligned}
& \frac{N_{0}}{-\mu}+\sum_{0<|m| \leqq \gamma} \frac{N_{m}}{\varepsilon N_{m}-\mu}+\frac{N_{0}}{\varepsilon N_{0}+\mu} \\
& +\sum_{0<|m| \leqq \gamma} \frac{N_{m}}{\varepsilon N_{0}+\varepsilon N_{m}+\mu}+1=0 .
\end{aligned}
$$


Then $I_{k}(\varepsilon)$ satisfies the inequality

$$
I_{k}(\varepsilon) \geqq \sum_{|m| \leqq \gamma} \beta_{m}-(1+\varepsilon) N_{0}-\sum_{0<|m| \leqq \gamma}\left[(1+\varepsilon) N_{m}+\varepsilon N_{0}\right]-N(\gamma) .
$$

Proof. Let $\lambda_{m},|m| \leqq \gamma$, be positive numbers such that $\lambda_{0} \leqq \lambda_{m}$ if $m \neq 0$. We define $A_{k, m}, B_{k, m}$ for $|m| \leqq \gamma$ by

$$
A_{k, m}=\lambda_{m}^{-1} T_{k, m}, \quad B_{k, m}=\lambda_{m}^{-1} S_{k, m} .
$$

It is easy to see that

$$
\begin{aligned}
I_{k}(\varepsilon) \geqq & \varepsilon\langle\psi| \sum_{0<|n| \leqq \gamma} \lambda_{n}^{2} A_{k, n}^{*} A_{k, n}+\lambda_{0}^{2} B_{k, 0}^{*} B_{k, 0} \\
& +\sum_{0<|n| \leqq \gamma}\left(\lambda_{0}^{2}+\lambda_{n}^{2}\right) B_{k, n}^{*} B_{k, n}|\psi\rangle \\
& +\left\langle\psi\left|S_{k}^{*} S_{k}+T_{k}^{*} T_{k}+S_{k} T_{k}+S_{k}^{*} T_{k}^{*}\right| \psi\right\rangle-\left|\left\langle\psi\left|S_{k}^{*}+T_{k}\right| \psi\right\rangle\right|^{2}
\end{aligned}
$$

Now let $C$ be the matrix $\lambda^{*} \lambda$, where $\lambda$ is the vector $\lambda=\left(\lambda_{0}, \ldots\right)$, and $D$ be the diagonal matrix with 0 in the first entry and $\varepsilon \lambda_{n}^{2}$ with $0<|n| \leqq \gamma$ in the other positions. From (4.21) and the commutation relations we see that

$$
\begin{aligned}
I_{k}(\varepsilon) \geqq & \left\langle\psi\left|\left[\begin{array}{l}
\mathbf{A}_{k} \\
\mathbf{B}_{k}^{*}
\end{array}\right]^{*}\left[\begin{array}{cc}
C+D & C \\
C & C+D+\varepsilon \lambda_{0}^{2} I
\end{array}\right]\left[\begin{array}{l}
\mathbf{A}_{k} \\
\mathbf{B}_{k}^{*}
\end{array}\right]\right| \psi\right\rangle \\
& -\left|\left\langle\psi\left|S_{k}^{*}+T_{k}\right| \psi\right\rangle\right|^{2}-\left\langle\psi\left|(1+\varepsilon)\left[a_{0}^{*} a_{0}-a_{k}^{*} a_{k}\right]\right| \psi\right\rangle \\
& -\left\langle\psi\left|\sum_{0<|m| \leqq \gamma}\left[(1+\varepsilon)+\varepsilon \lambda_{0}^{2} / \lambda_{m}^{2}\right]\left[a_{m}^{*} a_{m}-a_{m+k}^{*} a_{m+k}\right]\right| \psi\right\rangle,
\end{aligned}
$$

where $\mathbf{A}_{k}$ and $\mathbf{B}_{k}$ are the vectors, $\mathbf{A}_{k}=\left(A_{k, 0}, \ldots\right), \mathbf{B}_{k}=\left(B_{k, 0}, \ldots\right)$.

Next we choose a Bogoliubov matrix $M$ such that

$$
M^{*}\left[\begin{array}{cc}
C+D & C \\
C & C+D+\varepsilon \lambda_{0}^{2} I
\end{array}\right] M=\left[\begin{array}{cc}
\alpha_{0} & \\
& \beta_{0}
\end{array}\right] .
$$

It is certainly possible to do this since the matrix diagonalised in (4.23) is positive definite. The columns of $M$ are made up of vectors $(\mathbf{v}, \mathbf{w})$, where

$$
\begin{gathered}
C(\mathbf{v}+\mathbf{w})+D \mathbf{v}=\mu \mathbf{v}, \\
C(\mathbf{v}+\mathbf{w})+\left(D+\varepsilon \lambda_{0}^{2}\right) \mathbf{w}=-\mu \mathbf{w} .
\end{gathered}
$$

Evidently if $(\mathbf{v}, \mathbf{w})$ is a solution of $(4.24)$ with eigenvalue $\mu$ then $(\mathbf{w}, \mathbf{v})$ is also a solution with eigenvalue $-\mu-\varepsilon \lambda_{0}^{2}$. Thus on taking $\beta_{m}=\alpha_{m}+\varepsilon \lambda_{0}^{2},|m| \leqq \gamma$, we see that the matrix $M$ has the block form

$$
\left[\begin{array}{ll}
V & W \\
W & V
\end{array}\right]
$$

From (2.22) it follows that $V$ and $W$ satisfy the relations

$$
V^{*} V-W^{*} W=I: V^{*} W=W^{*} V
$$


It is then easy to see that

$$
M^{-1}=\left[\begin{array}{cc}
V^{*} & -W^{*} \\
-W^{*} & V^{*}
\end{array}\right] .
$$

We define a change of variables as in (2.21) by

$$
\left[\begin{array}{l}
\mathbf{A}_{k} \\
\mathbf{B}_{k}^{*}
\end{array}\right]=M\left[\begin{array}{l}
\boldsymbol{\eta}_{k} \\
\zeta_{k}^{*}
\end{array}\right] .
$$

where $\eta_{k}=\left(\eta_{k, 0}, \ldots\right)$ and $\zeta_{k}=\left(\zeta_{k, 0}, \ldots\right)$. In view of (4.23) we have from (4.22) the inequality

$$
\begin{aligned}
I_{k}(\varepsilon) \geqq & \left\langle\psi\left|\sum_{|m| \leqq \gamma} \alpha_{m} \eta_{k, m}^{*} \eta_{k, m}+\sum_{|m| \leqq \gamma} \beta_{m} \zeta_{k, m}^{*} \zeta_{k, m}\right| \psi\right\rangle \\
& -\left|\left\langle\psi\left|S_{k}^{*}+T_{k}\right| \psi\right\rangle\right|^{2}+\left\langle\psi\left|\sum_{|m| \leqq \gamma} \beta_{m}\left[\zeta_{k, m}, \zeta_{k, m}^{*}\right]\right| \psi\right\rangle \\
& -\left\langle\psi\left|(1+\varepsilon)\left[a_{0}^{*} a_{0}-a_{k}^{*} a_{k}\right]\right| \psi\right\rangle \\
& -\left\langle\psi\left|\sum_{0<|m| \leqq \gamma}\left[(1+\varepsilon)+\varepsilon \lambda_{0}^{2} / \lambda_{m}^{2}\right]\left[a_{m}^{*} a_{m}-a_{m+k}^{*} a_{m+k}\right]\right| \psi\right\rangle .
\end{aligned}
$$

We shall show that

$$
\left\langle\psi\left|\sum_{|m| \leqq \gamma} \alpha_{m} \eta_{k, m}^{*} \eta_{k, m}+\sum_{|m| \leqq \gamma} \beta_{m} \zeta_{k, m}^{*} \zeta_{k, m}\right| \psi\right\rangle-\left|\left\langle\psi\left|S_{k}^{*}+T_{k}\right| \psi\right\rangle\right|^{2} \geqq 0
$$

To do this note that

$$
S_{k}^{*}+T_{k}=\lambda \cdot(V+W) \boldsymbol{\eta}_{k}+\lambda \cdot(V+W) \zeta_{k}^{*}=\boldsymbol{v} \cdot \boldsymbol{\eta}_{k}+\boldsymbol{v} \cdot \zeta_{k}^{*},
$$

where $\boldsymbol{v}=(V+W)^{*} \lambda$. Thus

$$
\begin{aligned}
& \left|\left\langle\psi\left|S_{k}^{*}+T_{k}\right| \psi\right\rangle\right|^{2}=\left|\left\langle\psi\left|\boldsymbol{v} \cdot \boldsymbol{\eta}_{k}\right| \psi\right\rangle+\left\langle\psi\left|\boldsymbol{v} \cdot \zeta_{k}^{*}\right| \psi\right\rangle\right|^{2} \\
& \quad \leqq(1+\delta)\left|\left\langle\psi\left|\boldsymbol{v} \cdot \boldsymbol{\eta}_{k}\right| \psi\right\rangle\right|^{2}+(1+1 / \delta)\left|\left\langle\psi\left|\boldsymbol{v} \cdot \zeta_{k}^{*}\right| \psi\right\rangle\right|^{2},
\end{aligned}
$$

for any $\delta>0$. Let $A$ be the matrix defined by $A=v^{*} v$. Then it is clear that (4.30) holds provided there is a $\delta>0$ such that

$$
\begin{gathered}
(1+\delta) \xi A \xi \leqq \sum_{|m| \leqq \gamma} \alpha_{m} \xi_{m}^{2}, \\
(1+1 / \delta) \xi A \xi \leqq \sum_{|m| \leqq \gamma} \beta_{m} \xi_{m}^{2},
\end{gathered}
$$

for all real vectors $\xi=\left(\xi_{0}, \ldots\right)$.

Observe next that (4.33) holds provided

$$
\begin{aligned}
& (1+\delta)\left[\sum_{|m| \leqq \gamma} \frac{v_{m}^{2}}{\alpha_{m}}\right]^{2} \leqq \sum_{|m| \leqq \gamma} \frac{v_{m}^{2}}{\alpha_{m}}, \\
& (1+1 / \delta)\left[\sum_{|m| \leqq \gamma} \frac{v_{m}^{2}}{\beta_{m}}\right]^{2} \leqq \sum_{|m| \leqq \gamma} \frac{v_{m}^{2}}{\beta_{m}},
\end{aligned}
$$

where $\boldsymbol{v}=\left(v_{0}, \ldots\right)$. A necessary and sufficient condition for there to exist a $\delta$ satisfying (4.34) is

$$
\sum_{|m| \leqq \gamma} \frac{v_{m}^{2}}{\alpha_{m}}+\sum_{|m| \leqq \gamma} \frac{v_{m}^{2}}{\beta_{m}} \leqq 1 .
$$


We prove (4.35) in a similar fashion to how we proceeded in Sect. 2. Applying the transformation $M$ to the inequality

$$
\left[\begin{array}{ll}
C & C \\
C & C
\end{array}\right] \leqq\left[\begin{array}{cc}
C+D & C \\
C & C+D+\varepsilon \lambda_{0}^{2} I
\end{array}\right],
$$

we have

$$
\left[\begin{array}{ll}
A & A \\
A & A
\end{array}\right] \leqq\left[\begin{array}{ll}
\alpha_{0} \\
& \beta_{0}
\end{array}\right]
$$

Now apply the matrices in (4.37) to the vector $\left(\frac{v_{0}}{\alpha_{0}}, \ldots, \frac{t v_{0}}{\beta_{0}}, \ldots\right)$, where $t$ is a real parameter. Then we obtain

$$
\begin{aligned}
& {\left[\sum_{|m| \leqq \gamma} \frac{v_{m}^{2}}{\alpha_{m}}\right]^{2}+t^{2}\left[\sum_{|m| \leqq \gamma} \frac{v_{m}^{2}}{\beta_{m}}\right]^{2}+2 t\left[\sum_{|m| \leqq \gamma} \frac{v_{m}^{2}}{\alpha_{m}}\right]\left[\sum_{|m| \leqq \gamma} \frac{v_{m}^{2}}{\beta_{m}}\right]} \\
& \leqq \sum_{|m| \leqq \gamma} \frac{v_{m}^{2}}{\alpha_{m}}+t^{2} \sum_{|m| \leqq \gamma} \frac{v_{m}^{2}}{\beta_{m}} .
\end{aligned}
$$

Hence we have

$$
\begin{gathered}
\left\{\sum_{|m| \leqq \gamma} \frac{v_{m}^{2}}{\beta_{m}}-\left[\sum_{|m| \leqq \gamma} \frac{v_{m}^{2}}{\beta_{m}}\right]^{2}\right\} t^{2}-2 t\left[\sum_{|m| \leqq \gamma} \frac{v_{m}^{2}}{\alpha_{m}}\right]\left[\sum_{|m| \leqq \gamma} \frac{v_{m}^{2}}{\beta_{m}}\right] \\
+\sum_{|m| \leqq \gamma} \frac{v_{m}^{2}}{\alpha_{m}}-\left[\sum_{|m| \leqq \gamma} \frac{v_{m}^{2}}{\beta_{m}}\right]^{2} \geqq 0
\end{gathered}
$$

for all real values of $t$. The inequality (4.35) now easily follows on completing the square in (4.39).

Next we wish to compute the commutators $\left[\zeta_{k, m}, \zeta_{k, m}^{*}\right]$ in (4.29). In view of (4.27), (4.28) we have

$$
\zeta_{k}^{*}=-W^{*} \mathbf{A}_{k}+V^{*} \mathbf{B}_{k}^{*} .
$$

Hence

$$
\begin{aligned}
& {\left[\zeta_{k, m}, \zeta_{k, m}^{*}\right]} \\
& =\sum_{\substack{|n| \leqq \gamma \\
|j| \leqq \gamma}}\left[-W_{m, n}^{*} A_{k, n}^{*}+V_{m, n}^{*} B_{k, n},-W_{m, j}^{*} A_{k, j}+V_{m, j}^{*} B_{k, j}^{*}\right] \\
& =\sum_{|n| \leqq \gamma}\left\{W_{m, n}^{*} W_{m, n}^{*}\left[A_{k, n}^{*}, A_{k, n}\right]+V_{m, n}^{*} V_{m, n}^{*}\left[B_{k, n}, B_{k, n}^{*}\right]\right\} \\
& =\sum_{0<|n| \leqq \gamma}\left\{W_{m, n}^{*} W_{m, n}^{*} \lambda_{n}^{-2}\left[a_{n-k}^{*} a_{n-k}-a_{n}^{*} a_{n}\right]\right. \\
& \left.\quad+V_{m, n}^{*} V_{m, n}^{*} \lambda_{n}^{-2}\left[a_{n}^{*} a_{n}-a_{n+k}^{*} a_{n+k}\right]\right\} \\
& \quad+W_{m, 0}^{*} W_{m, 0}^{*} \lambda_{0}^{-2}\left[a_{-k}^{*} a_{-k}-a_{0}^{*} a_{0}+\sum_{\{n:|n|>\gamma,|n+k|>\gamma\}}\right. \\
& \left.\quad \cdot\left\{a_{n}^{*} a_{n}-a_{n+k}^{*} a_{n+k}\right\}\right]+V_{m, 0}^{*} V_{m, 0}^{*} \lambda_{0}^{-2}\left[a_{0}^{*} a_{0}-a_{k}^{*} a_{k}\right] .
\end{aligned}
$$


Now let us define $J_{k}(\varepsilon)$ by

$$
\begin{aligned}
J_{k}(\varepsilon)= & \langle\psi| \sum_{|m| \leqq \gamma} \beta_{m} \sum_{|n| \leqq \gamma}\left[V_{m, n}^{*} V_{m, n}^{*}-W_{m, n}^{*} W_{m, n}^{*}\right] \\
& \cdot \lambda_{n}^{-2} a_{n}^{*} a_{n}|\psi\rangle-\left\langle\psi\left|(1+\varepsilon) a_{0}^{*} a_{0}\right| \psi\right\rangle \\
& -\left\langle\psi\left|\sum_{0<|m| \leqq \gamma}\left[(1+\varepsilon)+\varepsilon \lambda_{0}^{2} / \lambda_{m}^{2}\right] a_{m}^{*} a_{m}\right| \psi\right\rangle,
\end{aligned}
$$

and $H_{k}(\varepsilon)$ by

$$
\begin{aligned}
H_{k}(\varepsilon)= & \sum_{|m| \leqq \gamma} \beta_{m}\langle\psi| \sum_{0<|n| \leqq \gamma} \lambda_{n}^{-2}\left[W_{m, n}^{*} W_{m, n}^{*} a_{n-k}^{*} a_{n-k}\right. \\
& \left.-V_{m, n}^{*} V_{m, n}^{*} a_{n+k}^{*} a_{n+k}\right]+W_{m, 0}^{*} W_{m, 0}^{*} \lambda_{0}^{-2} \\
& \cdot\left[a_{-k}^{*} a_{-k}+\sum_{\{n:|n|>\gamma,|n+k|>\gamma\}}\left\{a_{n}^{*} a_{n}-a_{n+k}^{*} a_{n+k}\right\}\right] \\
& -V_{m, 0}^{*} V_{m, 0}^{*} \lambda_{0}^{-2} a_{k}^{*} a_{k}|\psi\rangle+\left\langle\psi\left|(1+\varepsilon) a_{k}^{*} a_{k}\right| \psi\right\rangle \\
& +\left\langle\psi\left|\sum_{0<|m| \leqq \gamma}\left[(1+\varepsilon)+\varepsilon \lambda_{0}^{2} / \lambda_{m}^{2}\right] a_{m+k}^{*} a_{m+k}\right| \psi\right\rangle .
\end{aligned}
$$

It is clear from (4.29) and (4.41) that

$$
I_{k}(\varepsilon) \geqq J_{k}(\varepsilon)+H_{k}(\varepsilon) .
$$

We may simplify the expression for $H_{k}(\varepsilon)$ a little by noting that

$$
\sum_{\{n:|n|>\gamma,|n+k|>\gamma\}}\left[a_{n}^{*} a_{n}-a_{n+k}^{*} a_{n+k}\right]=\sum_{|n| \leqq \gamma}\left[a_{n+k}^{*} a_{n+k}-a_{n-k}^{*} a_{n-k}\right] .
$$

Thus we have

$$
\begin{aligned}
H_{k}(\varepsilon)= & \sum_{0<|n| \leqq \gamma}\left\langle\psi\left|a_{n-k}^{*} a_{n-k}\right| \psi\right\rangle \cdot\left[\sum_{|m| \leqq \gamma} \beta_{m}\left\{\lambda_{n}^{-2}\left(W_{n, m}\right)^{2}-\lambda_{0}^{-2}\left(W_{0, m}\right)^{2}\right\}\right] \\
& +\sum_{0<|n| \leqq \gamma}\left\langle\psi\left|a_{n+k}^{*} a_{n+k}\right| \psi\right\rangle\left[1+\varepsilon+\varepsilon \lambda_{0}^{2} / \lambda_{n}^{2}\right. \\
& \left.+\sum_{|m| \leqq \gamma} \beta_{m}\left\{\lambda_{0}^{-2}\left(W_{0, m}\right)^{2}-\lambda_{n}^{-2}\left(V_{n, m}\right)^{2}\right\}\right] \\
& +\left\langle\psi\left|a_{k}^{*} a_{k}\right| \psi\right\rangle\left[1+\varepsilon+\sum_{|m| \leqq \gamma} \beta_{m}\left\{\lambda_{0}^{-2}\left(W_{0, m}\right)^{2}-\lambda_{0}^{-2}\left(V_{0, m}\right)^{2}\right\}\right] .
\end{aligned}
$$

We shall bound $H_{k}(\varepsilon)$ from below. To do this first note that, in view of the formula (4.27) for $M^{-1}$, we have from the identity $M M^{-1}=I$ the equalities

$$
V V^{*}-W W^{*}=I ; \quad V W^{*}=W V^{*} .
$$

From (4.47) we see that

$$
\sum_{|m| \leqq \gamma}\left[\left(V_{0, m}\right)^{2}-\left(W_{0, m}\right)^{2}\right]=1 .
$$

It follows therefore that

$$
\begin{aligned}
1+ & \varepsilon+\sum_{|m| \leqq \gamma} \beta_{m} \lambda_{0}^{-2}\left\{\left(W_{0, m}\right)^{2}-\left(V_{0, m}\right)^{2}\right\} \\
& =1+\varepsilon+\sum_{|m| \leqq \gamma}\left(\alpha_{m}+\varepsilon \lambda_{0}^{2}\right) \lambda_{0}^{-2}\left\{\left(W_{0, m}\right)^{2}-\left(V_{0, m}\right)^{2}\right\} \\
& \geqq 1-\lambda_{0}^{-2} \sum_{|m| \leqq \gamma} \alpha_{m}\left(V_{0, m}\right)^{2} .
\end{aligned}
$$


If we multiply (4.23) on the left and the right by $\left(M^{*}\right)^{-1}, M^{-1}$ respectively and use the formula (4.27), we obtain the identity

$$
V\left[{ }^{\alpha_{0}} \cdot\right] V^{*}+W\left[\beta_{0} \cdot\right] W^{*}=C+D .
$$

On evaluating the first entry in the matrix (4.50) we easily obtain the inequality

$$
\sum_{|m| \leqq \gamma} \alpha_{m}\left(V_{0, m}\right)^{2} \leqq \lambda_{0}^{2} .
$$

It follows then from (4.49) that the coefficient of $\left\langle\psi\left|a_{k}^{*} a_{k}\right| \psi\right\rangle$ in (4.46) is nonnegative.

By an exactly similar argument we see that for $0<|n| \leqq \gamma$ the coefficient of $\left\langle\psi\left|a_{n+k}^{*} a_{n+k}\right| \psi\right\rangle$ in (4.46) is bounded below by

$$
\begin{gathered}
\varepsilon \lambda_{0}^{2} / \lambda_{n}^{2}+\sum_{|m| \leqq \gamma} \varepsilon \lambda_{0}^{2}\left\{\lambda_{0}^{-2}\left(W_{0, m}\right)^{2}-\lambda_{n}^{-2}\left(V_{n, m}\right)^{2}\right\} \\
\geqq \sum_{|m| \leqq \gamma} \varepsilon \lambda_{0}^{2}\left\{\lambda_{0}^{-2}\left(W_{0, m}\right)^{2}-\lambda_{n}^{-2}\left(W_{n, m}\right)^{2}\right\} .
\end{gathered}
$$

Observe next that $W_{0, m}$ and $W_{n, m}$ with $0<|n| \leqq \gamma$ satisfy according to (4.24) the equations

$$
v_{m} \lambda_{0}=-\beta_{m} W_{0, m} ; \quad v_{m} \lambda_{n}=-\left(\beta_{m}+\varepsilon \lambda_{n}^{2}\right) W_{n, m} .
$$

Hence the last expression in (4.52) is non-negative. Thus the coefficient of $\left\langle\psi\left|a_{n+k}^{*} a_{n+k}\right| \psi\right\rangle$ in (4.46) is non-negative for $0<|n| \leqq \gamma$.

The coefficient of $\left\langle\psi\left|a_{n-k}^{*} a_{n-k}\right| \psi\right\rangle$ in (4.46) is bounded below by

$$
-\lambda_{0}^{-2} \sum_{|m| \leqq \gamma} \beta_{m}\left(W_{0, m}\right)^{2} \geqq-\sum_{|m| \leqq \gamma} \frac{v_{m}^{2}}{\beta_{m}} \geqq-1,
$$

from (4.35). We conclude therefore that $H_{k}(\varepsilon)$ satisfies the inequality

$$
H_{k}(\varepsilon) \geqq-N(\gamma) \text {. }
$$

To conclude the lemma we let $\lambda_{n}^{2} \rightarrow\left\langle\psi\left|a_{n}^{*} a_{n}\right| \psi\right\rangle$ for $|n| \leqq \gamma$. In view of the fact that

$$
\sum_{|n| \leqq \gamma}\left[\left(V_{n, m}\right)^{2}-\left(W_{n, m}\right)^{2}\right]=1
$$

we see that $J_{k}(\varepsilon)$ is bounded below by the expression on the right-hand side of (4.19). Q.E.D.

Next we wish to estimate from below the quantity on the right of (4.19). To do this we prove a lemma similar to Lemma 2.1 .

Lemma 4.2. Let $\lambda_{n}, n=0,1,2, \ldots$ be real numbers and $p$ be a positive number with $p<1$. Suppose $c_{n}$ are non-negative numbers such that

$$
M_{1} n^{p} \leqq c_{n} \leqq M_{2} n^{p}, \quad n=0,1,2, \ldots,
$$


for some positive constants $M_{1}$ and $M_{2}$. Define $N, K, n_{0}$ by

$$
\begin{gathered}
N=\sum_{n=0}^{\infty} \lambda_{n}^{2} ; \quad K=\sum_{n=0}^{\infty} c_{n} \lambda_{n}^{2}, \\
n_{0}=\left[(K / N)^{1 / p}\right]+1 .
\end{gathered}
$$

Thus $n_{0}$ is an integer and $n_{0} \geqq 1$.

Suppose now that only a finite number of the $\lambda_{n}$ are non-zero and that $\lambda_{m}$ is the smallest of these in absolute value. Let $\alpha_{n}, n=0,1,2, \ldots$, be the non-negative zeros of the equation

$$
\frac{\lambda_{m}^{2}}{-\mu}+\sum_{n \neq m} \frac{\lambda_{n}^{2}}{\varepsilon \lambda_{n}^{2}-\mu}+\frac{\lambda_{m}^{2}}{\varepsilon \lambda_{m}^{2}+\mu}+\sum_{n \neq m} \frac{\lambda_{n}^{2}}{\varepsilon \lambda_{n}^{2}+\varepsilon \lambda_{m}^{2}+\mu}+1=0,
$$

and $I(\varepsilon)$ be defined for $\varepsilon>0$ by

$$
I(\varepsilon)=\sum_{n=0}^{\infty} \alpha_{n}-\lambda_{m}^{2}-\sum_{n \neq m}(1+\varepsilon) \lambda_{n}^{2} .
$$

Then $I(\varepsilon)$ satisfies the inequalities

$$
\begin{gathered}
I(\varepsilon) \geqq-N, \\
I(\varepsilon) \geqq-C N\left(n_{0} / \varepsilon\right)^{p} \quad \text { if } \quad \varepsilon>n_{0},
\end{gathered}
$$

where $C$ is a constant depending only on $p, M_{1}, M_{2}$.

Proof. The inequality (4.62) follows since we can find zeros $\alpha_{n}$ with $\alpha_{n} \geqq \varepsilon \lambda_{n}^{2}$ for $n \neq m$.

We turn to (4.63). Let $\alpha_{n}^{(1)}$ be the non-negative zeros of the equation

$$
\frac{\lambda_{m}^{2}}{-\mu}+\sum_{n \neq m} \frac{\lambda_{n}^{2}}{\varepsilon \lambda_{n}^{2}-\mu}+\frac{\lambda_{m}^{2}}{\varepsilon \lambda_{m}^{2}+\mu}+\sum_{n \neq m} \frac{\lambda_{n}^{2}}{\varepsilon \lambda_{n}^{2}+\mu}+1=0 .
$$

Arguing just as in Sect. 2 we see that

$$
\sum_{n=0}^{\infty} \alpha_{n} \geqq \sum_{n=0}^{\infty} \alpha_{n}^{(1)}
$$

If we let $-\beta_{n}^{(1)}, n=0,1, \ldots$, be the negative roots of (4.64) then we have

and hence that

$$
\sum_{n=0}^{\infty} \alpha_{n}^{(1)}-\sum_{n=0}^{\infty} \beta_{n}^{(1)}=-\varepsilon \lambda_{m}^{2}
$$

$$
I(\varepsilon) \geqq \sum_{n=0}^{\infty} \beta_{n}^{(1)}-\sum_{n=0}^{\infty}(1+\varepsilon) \lambda_{n}^{2}
$$

Now the $\beta_{n}^{(1)}$ are the positive zeros of the equation

$$
\sum_{n=0}^{\infty} \frac{\lambda_{n}^{2}}{\varepsilon \lambda_{n}^{2}-\mu}+\sum_{n=0}^{\infty} \frac{\lambda_{n}^{2}}{\varepsilon \lambda_{n}^{2}+\mu}+\left[\frac{\lambda_{m}^{2}}{\mu}-\frac{\lambda_{m}^{2}}{\varepsilon \lambda_{m}^{2}+\mu}\right]+1=0 .
$$

The expression in square brackets in (4.68) is

$$
\varepsilon \lambda_{m}^{4} / \mu\left(\varepsilon \lambda_{m}^{2}+\mu\right)
$$


In view of our definition of $\lambda_{m}$, it follows that all the positive zeros $\mu$ of (4.68) satisfy $\mu \geqq \varepsilon \lambda_{m}^{2}$. Furthermore, if $\mu \geqq \varepsilon \lambda_{m}^{2}$, then the expression (4.69) is bounded above by $1 / 2 \varepsilon$. Thus if we let $\alpha_{n}^{(2)}$ be the positive zeros of the equation

$$
\sum_{n=0}^{\infty} \frac{\lambda_{n}^{2}}{\varepsilon \lambda_{n}^{2}-\mu}+\sum_{n=0}^{\infty} \frac{\lambda_{n}^{2}}{\varepsilon \lambda_{n}^{2}+\mu}+1+\frac{1}{2 \varepsilon}=0,
$$

then we have

$$
I(\varepsilon) \geqq \sum_{n=0}^{\infty} \alpha_{n}^{(2)}-\sum_{n=0}^{\infty}(1+\varepsilon) \lambda_{n}^{2} .
$$

Next we define for $k=0,1,2, \ldots$,

$$
N_{k}=\sum_{\left(2^{k}-1\right) n_{0}}^{\left(2^{k+1}-1\right) n_{0}-1} \lambda_{n}^{2} .
$$

It is clear from (4.57) and (4.58) that we may find a constant $C$ such that

$$
N_{k} \leqq C N / 2^{k p}, \quad k \geqq 0 .
$$

Let $\alpha_{n}^{(3)}$ be the positive zeros of the equation

$$
\sum_{n=0}^{\infty} \frac{\lambda_{n}^{2}}{\varepsilon \lambda_{n}^{2}-\mu}+\sum_{k=0}^{\infty} \frac{N_{k}}{\varepsilon N_{k} / 2^{k} n_{0}+\mu}+1+\frac{1}{2 \varepsilon}=0 .
$$

Then we have

$$
\sum_{n=0}^{\infty} \alpha_{n}^{(2)} \geqq \sum_{n=0}^{\infty} \alpha_{n}^{(3)}
$$

Letting $-\beta_{k}^{(3)}$ be the negative zeros of (4.74) we see that

$$
\begin{aligned}
& \sum_{n=0}^{\infty} \alpha_{n}^{(3)}-\sum_{k=0}^{\infty} \beta_{k}^{(3)} \\
& \quad=\left[(1+1 / 2 \varepsilon)^{-1}+\varepsilon\right] \sum_{n=0}^{\infty} \lambda_{n}^{2}-\sum_{k=0}^{\infty}\left[(1+1 / 2 \varepsilon)^{-1}+\varepsilon / 2^{k} n_{0}\right] N_{k} .
\end{aligned}
$$

From (4.71) it then follows that

$$
I(\varepsilon) \geqq \sum_{k=0}^{\infty} \beta_{k}^{(3)}-\sum_{k=0}^{\infty}\left[(1+1 / 2 \varepsilon)^{-1}+\varepsilon / 2^{k} n_{0}\right] N_{k}-N / 2 \varepsilon .
$$

Arguing as before we see that if $\alpha_{k}^{(4)}$ are the positive zeros of the equation

$$
\sum_{k=0}^{\infty} \frac{N_{k}}{\varepsilon N_{k} / 2^{k} n_{0}-\mu}+\sum_{k=0}^{\infty} \frac{N_{k}}{\varepsilon N_{k} / 2^{k} n_{0}+\mu}+1+\frac{1}{2 \varepsilon}=0,
$$

then $I(\varepsilon)$ satisfies the inequality

$$
I(\varepsilon) \geqq \sum_{k=0}^{\infty} \alpha_{k}^{(4)}-\sum_{k=0}^{\infty}\left[(1+1 / 2 \varepsilon)^{-1}+\varepsilon / 2^{k} n_{0}\right] N_{k}-N / 2 \varepsilon .
$$

It is easy to argue further that the right side of (4.79) is minimized when the $N_{k}$ take on their maximum values as given by (4.73). Hence we are in the following 
situation: Let $\eta_{k}=C / 2^{k p}, k \geqq 0$, where $C$ is the constant in (4.73) and $\delta=\varepsilon / n_{0}$. Then $I(\varepsilon)$ satisfies the inequality

$$
\frac{I(\varepsilon)}{N} \geqq \sum_{k=0}^{\infty} \gamma_{k}-\sum_{k=0}^{\infty}\left[(1+1 / 2 \varepsilon)^{-1}+\delta / 2^{k}\right] \eta_{k}-1 / 2 \varepsilon
$$

where the $\gamma_{k}$ are the positive zeros of the equation

$$
\sum_{k=0}^{\infty} \frac{\eta_{k}}{\delta \eta_{k} / 2^{k}-\mu}+\sum_{k=0}^{\infty} \frac{\eta_{k}}{\delta \eta_{k} / 2^{k}+\mu}+1+\frac{1}{2 \varepsilon}=0 .
$$

Let $\gamma_{k}$ be the unique zero of (4.81) such that $\delta \eta_{k-1} / 2^{k-1}>\gamma_{k}>\delta \eta_{k} / 2^{k}$. We bound the zeros $\gamma_{k}$ below as follows: If $\delta \leqq 2^{k}$ bound $\gamma_{k}$ below by

$$
\gamma_{k}>\delta \eta_{k} / 2^{k}
$$

If $\delta>2^{k}$ we proceed differently. In that case we have

$$
\sum_{m=0}^{\infty} \frac{\eta_{m}}{\delta \eta_{m} / 2^{m}+\gamma_{k}}=\sum_{m<k}+\sum_{m \geqq k}
$$

Since we have

$$
\begin{gathered}
\sum_{m<k} \leqq \sum_{m<k} \frac{2^{m}}{\delta}<\frac{2^{k}}{\delta}, \\
\sum_{m \geqq k} \leqq \sum_{m \geqq k} \frac{\eta_{m}}{\gamma_{k}} \leqq \frac{C_{1} \eta_{k}}{\gamma_{k}}<\frac{C_{1} 2^{k}}{\delta}
\end{gathered}
$$

for some constant $C_{1}$ depending on $C$ and $p$, it follows that there is constant $C_{2}$ such that

$$
\sum_{m=0}^{\infty} \frac{\eta_{m}}{\delta \eta_{m} / 2^{m}+\gamma_{k}} \leqq C_{2} \frac{2^{k}}{\delta}
$$

We may also see that

$$
\sum_{m<k-1} \frac{\eta_{m}}{\delta \eta_{m} / 2^{m}-\gamma_{k}} \leqq \sum_{m<k-1} \frac{\eta_{m}}{\delta \eta_{m} / 2^{m}-\delta \eta_{k-1} / 2^{k-1}} \leqq \frac{C_{3} 2^{k}}{\delta},
$$

for some constant $C_{3}$. Hence there is a constant $C_{4}$ such that

$$
\frac{\eta_{k-1}}{\delta \eta_{k-1} / 2^{k-1}-\gamma_{k}}+\frac{\eta_{k}}{\delta \eta_{k} / 2^{k}-\gamma_{k}}+C_{4} \frac{2^{k}}{\delta}+1 \geqq 0,
$$

in view of the fact that $n_{0} \geqq 1$. Then $\gamma_{k} \geqq \gamma_{k}^{\prime}$, where $\gamma_{k}^{\prime}$ is the unique zero of the equation

$$
\frac{\eta_{k-1}}{\delta \eta_{k-1} / 2^{k-1}-\mu}+\frac{\eta_{k}}{\delta \eta_{k} / 2^{k}-\mu}+C_{4} \frac{2^{k}}{\delta}+1=0
$$

which has $\delta \eta_{k-1} / 2^{k-1}>\mu>\delta \eta_{k} / 2^{k}$. It is not difficult to see from (4.89) that there is a constant $C_{5}$ with

$$
\gamma_{k}^{\prime} \geqq \delta \eta_{k} / 2^{k}+\eta_{k}\left[C_{5} \frac{2^{k}}{\delta}+1\right]^{-1}
$$

We bound $\gamma_{k}$ below by the right side of (4.90) in the case when $\delta>2^{k}$. 
We are now in a position to estimate the right side of (4.80). We write it as

$$
\sum_{k=0}^{\infty}\left[\gamma_{k}-(1+1 / 2 \varepsilon)^{-1} \eta_{k}-\delta \eta_{k} / 2^{k}\right]-1 / 2 \varepsilon=\sum_{\delta \leqq 2^{k}}+\sum_{\delta>2^{k}}-1 / 2 \varepsilon
$$

From (4.82) we have

$$
\sum_{\delta \leqq 2^{k}} \geqq-(1+1 / 2 \varepsilon)^{-1} \sum_{\delta \leqq 2^{k}} \eta_{k} \geqq-C_{6} / \delta^{p},
$$

for some constant $C_{6}$ if we assume $\varepsilon>n_{0}$ and hence $\varepsilon>1$. From (4.90) we see that

$$
\sum_{\delta>2^{k}} \geqq-C_{7} / \delta^{p},
$$

in a similar fashion. Hence since $\delta=\varepsilon / n_{0}$ we have from (4.80) that

$$
\frac{I(\varepsilon)}{N} \geqq-C\left(n_{0} / \varepsilon\right)^{p},
$$

provided $\varepsilon>n_{0}$. We are finished. Q.E.D

We wish now to apply the previous two lemmas to our problem. Let $N_{k},|k| \leqq \gamma$ be the same as in Lemma 4.1 and put

$$
N^{\prime}=\sum_{|k| \leqq \gamma} N_{k} ; \quad K^{\prime}=\sum_{|k| \leqq \gamma} k^{2} N_{k} .
$$

If we define $n_{0}$ as in (4.59) with $N$ replaced by $N^{\prime}$ and $K$ by $K^{\prime}$ we see from Lemmas 4.1 and 4.2 that $I_{k}(\varepsilon)$ satisfies the inequalities

$$
\begin{gathered}
I_{k}(\varepsilon) \geqq-N^{\prime}-N(\gamma), \\
I_{k}(\varepsilon) \geqq-C N^{\prime}\left(n_{0} / \varepsilon\right)^{2 / 3}-N(\gamma), \quad \varepsilon>n_{0},
\end{gathered}
$$

where $C$ is a universal constant.

To finish our proof we need to prove $(1.15)$ with the Coulomb potential replaced by the periodic potential $V_{\alpha}(x)$. From the estimates (4.7) and (4.8) we have

$$
\begin{aligned}
& \frac{N^{\alpha}}{\varrho^{1 / 3}}\left\langle\psi, K_{N} \psi\right\rangle+\mathscr{E}_{V_{\alpha}}\left(|\psi|^{2}\right) \geqq \frac{N^{\alpha}}{2 \varrho^{1 / 3}} \int(\nabla \sqrt{\varrho})^{2} d x+C_{1} N^{\alpha-2 / 3} K^{\prime} \varrho^{1 / 3} \\
& \quad-C_{2} \sum_{|k|>2 \gamma} \frac{N^{-1 / 3} \varrho^{1 / 3}}{k^{2}+1} I_{k}\left(N^{\alpha-4 / 3} k^{4}\right)-C_{3} N^{7 / 5-\alpha} \varrho^{1 / 3},
\end{aligned}
$$

where $C_{1}, C_{2}$, and $C_{3}$ are universal constants. We can estimate the sum in (4.98) by using (4.96) and (4.97). Thus we have

$$
C_{2} \sum_{|k|>2 \gamma} \frac{N^{-1 / 3} \varrho^{1 / 3}}{k^{2}+1} I_{k}\left(N^{\alpha-4 / 3} k^{4}\right) \leqq C_{4} N^{\prime} N^{-\alpha / 4} n_{0}^{1 / 4} \varrho^{1 / 3} .
$$

Observe next that provided $n_{0} \geqq 2$, we have

$$
C_{1} N^{\alpha-2 / 3} K^{\prime} \varrho^{1 / 3} \geqq C_{5} N^{\prime} N^{\alpha-2 / 3} n_{0}^{2 / 3} \varrho^{1 / 3} .
$$

It is easy to see that

$$
C_{5} N^{\alpha-2 / 3} n_{0}^{2 / 3}-C_{4} N^{-\alpha / 4} n_{0}^{1 / 4} \geqq C_{6} N^{2 / 5-\alpha},
$$


for some universal constant $C_{6}$. In view of the fact that $N^{\prime} \leqq N$ we are done in the case $n_{0} \geqq 2$. If $n_{0}=1$ we use Lemma 3.1. Hence we have

$$
\frac{N^{\alpha}}{2 \varrho^{1 / 3}} \int(\nabla \sqrt{\varrho})^{2} d x \geqq C_{7} N^{\alpha+1 / 3} \varrho^{1 / 3} \geqq C_{7} N^{\prime} N^{\alpha-2 / 3} n_{0}^{2 / 3} \varrho^{1 / 3} .
$$

Therefore we can apply (4.101) again. Hence we have proved Theorem 1.4 in the case when $\alpha \leqq 8 / 15$.

For $\alpha>8 / 15$ we choose $\gamma$ such that

$$
\begin{gathered}
N(\gamma) \sum_{k \in Z^{3}} v_{\alpha}(k) \leqq C N^{1 / 3+\alpha} \varrho^{1 / 3}, \\
N \sum_{|k| \leqq 2 \gamma} v_{\alpha}(k) \leqq C N^{1 / 3+\alpha} \varrho^{1 / 3},
\end{gathered}
$$

where $C$ is a suitably small universal constant. From (3.44) we have that

$$
\begin{gathered}
\sum_{k} v_{\alpha}(k) \leqq C_{1} N^{1 / 3-\alpha / 2} \varrho^{1 / 3}, \\
N \sum_{|k| \leqq 2 \gamma} v_{\alpha}(k) \leqq C_{1} N^{2 / 3} \gamma \varrho^{1 / 3},
\end{gathered}
$$

for some constant $C_{1}$ independent of $N$. Arguing as before we may assume that $N(\gamma)$ satisfies the inequality (4.13). Hence we need $\gamma$ such that $\gamma=N^{r}$ with

$$
\frac{5}{6}-\frac{5 \alpha}{4}<r<\alpha-1 / 3
$$

Such a choice for $\gamma$ is clearly possible when $\alpha \geqq 8 / 15$. The rest of the argument in the proof of Theorem 1.4 then follows in exactly the same way as in the case $\alpha<8 / 15$.

Hence the proof of Theorem 1.4 is complete.

Remark. Observe that there is no loss of generality in the restriction $N_{0} \leqq N_{k}$ for all $k$ with $|k| \leqq \gamma$ in Lemma 4.1. It is simply related to the fact that we defined $T_{k, 0}$ differently to the $T_{k, m}$ with $m \neq 0$.

We complete this section by estimating an asymptotic value for the constant $C(b)$ of Theorem 1.1 as $N \rightarrow \infty$. In Lemma 4.2 we take $p=2 / 3$ and the $c_{n}$ as the $k^{2}$ from (4.95). We obtain the value $C=75$ in the inequality (4.63). Using this value of $C$ we may estimate the constant $C_{4}$ in (4.101) as $C_{4}=8.8$. The constant $C_{5}$ in (4.101) turns out to be $C_{5}=2.47$. When we minimize (4.101) with respect to $n_{0}$ we obtain $C_{6}=-6.5$. Since the correction terms estimated in Sect. 3 are of lower order in $N$ than $N^{7 / 5}$ we may take $C(b)$ to be 6.5 asymptotically in $N$.

\section{Achieving the Lower Bound}

In this section we show that the lower bound obtained in Theorem 1.4 is sharp provided $\alpha<8 / 15$. It is also sharp when $\alpha=8 / 15$ provided one replaces the coefficient $N^{\alpha} / \varrho^{1 / 3}$ of the kinetic energy in (1.15) by a suitably small constant times $N^{\alpha} / \varrho^{1 / 3}$. Dyson's construction [3] corresponds to the case $\alpha=8 / 15$. 
Theorem 5.1. Let $\delta>0$ and $\alpha<8 / 15-\delta$. Suppose $\Lambda_{N}$ are a sequence of cubes $N=1,2, \ldots$ such that $N / \operatorname{vol} \Lambda_{N}=\varrho$ is fixed as $N \rightarrow \infty$. Then there exist constants $C_{\delta}$, $b_{\delta}$ and wave functions $\psi_{\alpha, N}\left(x_{1}, \ldots, x_{N}\right)$ such that the following hold:

(a) $\psi_{\alpha, N}$ is supported in $\left(\Lambda_{N}\right)^{N}$ and takes on the value zero on the boundary.

(b) $\psi_{\alpha, N}$ satisfies the inequality

$$
\frac{N^{\alpha}}{\varrho^{1 / 3}}\left\langle\psi_{\alpha, N}, K_{N} \psi_{\alpha, N}\right\rangle+\mathscr{E}\left(\left|\psi_{\alpha, N}\right|^{2}\right) \leqq-C_{\delta} N^{7 / 5-\alpha} \varrho^{1 / 3}
$$

To prove Theorem 5.1 we rigorise the heuristics discussed in Sect. 2. First we need to construct the wave function $\psi$ mentioned in Bogoliubov's principle which minimizes $J(\psi)$ at least cost of kinetic energy. In (2.20) we take $\lambda_{j}^{2}=N / n$ and $\varepsilon=\beta n$. Thus we can construct a wave function $\psi$ such that

$$
J(\psi)+N \beta \sum_{i=1}^{n}\left\langle\psi\left|a_{i}^{*} a_{i}+b_{i}^{*} b_{i}\right| \psi\right\rangle=N\left[\left(\beta^{2}+2 \beta\right)^{1 / 2}-1-\beta\right],
$$

and this $|\psi\rangle$ is just the vacuum state for the Boson operators $\eta_{i}, \zeta_{i}, 1 \leqq i \leqq n$. We wish to evaluate $\left\langle\psi\left|a_{i}^{*} a_{i}\right| \psi\right\rangle,\left\langle\psi\left|b_{i}^{*} b_{i}\right| \psi\right\rangle$ and to express $|\psi\rangle$ in terms of the vacuum $|0\rangle$ for the $a_{i}, b_{i}, 1 \leqq i \leqq n$.

In order to accomplish this we need to calculate the matrix $M$ defined in (2.21). Let us suppose in Eq. (2.34) that $\lambda_{i}^{2}=\lambda^{2}, i=1,2, \ldots, n$. Then (2.34) has $n-1$ positive zeros $\mu=\varepsilon \lambda^{2}$ and an $n^{\text {th }}$ zero larger than $\varepsilon \lambda^{2}$. When $\mu=\varepsilon \lambda^{2}$ the corresponding solutions $\mathbf{v}, \mathbf{w}$ of $(2.30)$ have $\mathbf{w}=0$ and $\mathbf{v}=[1,1, \ldots, 1]$. We may take the $n-1$ vectors $\left[\mathbf{v}_{j}, \mathbf{w}_{j}\right], 1 \leqq j \leqq n-1$, corresponding to the $n-1$ fold zero $\mu=\varepsilon \lambda^{2}$ as

$$
\mathbf{w}_{j}=0 ; \quad \mathbf{v}_{j}=\left(j^{2}+j\right)^{-1 / 2}[1,1, \ldots, 1,-j, 0, \ldots, 0] .
$$

The entries in the vector $\mathbf{v}_{j}$ are 1 in the first $j$ positions, $-j$ in the $(j+1)^{\text {st }}$ position and 0 otherwise. It is easy to see that the $\mathbf{v}_{j} 1 \leqq j \leqq n-1$, form an orthonormal set of vectors at right angles to $[1,1, \ldots, 1]$.

Let $\mu_{n}$ be the $n^{\text {th }}$ positive solution of (2.34) with corresponding vectors $\mathbf{v}_{n}, \mathbf{w}_{n}$. It is evident that $\mathbf{v}_{n}$ and $\mathbf{w}_{n}$ must be parallel to the vector $[1,1, \ldots, 1]$. Hence we may write

$$
\mathbf{w}_{n}=-n^{-1 / 2} \sinh \theta[1,1, \ldots, 1] ; \quad \mathbf{v}_{n}=n^{-1 / 2} \cosh \theta[1,1, \ldots, 1],
$$

where $\theta$ depends on $\varepsilon=\beta n$. Thus $\left[\mathbf{v}_{n}, \mathbf{w}_{n}\right]$ satisfy the normalization condition (2.29). From (2.30) one can see that $\theta$ is given in terms of $\beta$ by the equation

$$
\tanh 2 \theta=1 /(1+\beta)
$$

Since we may now construct the matrix $M$ we can see that

$$
\left\langle\psi\left|a_{i}^{*} a_{i}\right| \psi\right\rangle=\left\langle\psi\left|b_{i}^{*} b_{i}\right| \psi\right\rangle=\frac{\sinh ^{2} \theta}{n} .
$$

Thus for fixed $\beta$ the number of particles in the $a_{i}$ and $b_{i}$ states is proportional to $1 / n$. 
Next we construct $|\psi\rangle$ in terms of the vacuum $|0\rangle$ for the $a_{i}, b_{i}$. To do this observe that the $\eta_{j}, \zeta_{j}, 1 \leqq j \leqq n-1$ are given by

$$
\begin{aligned}
& \eta_{j}=\left(j^{2}+j\right)^{-1 / 2}\left[\sum_{i=1}^{j} a_{i}-j a_{j+1}\right], \\
& \zeta_{j}=\left(j^{2}+j\right)^{-1 / 2}\left[\sum_{i=1}^{j} b_{i}-j b_{j+1}\right] .
\end{aligned}
$$

Furthermore, if we define operators $a$ and $b$ by

$$
a=n^{-1 / 2} \sum_{i=1}^{n} a_{i}, \quad b=n^{-1 / 2} \sum_{i=1}^{n} b_{i},
$$

then one can see that $a, b$ are related to $\eta_{n}, \zeta_{n}$ by the equations

$$
\begin{gathered}
a=\cosh \theta \eta_{n}-\sinh \theta \zeta_{n}^{*}, \\
b^{*}=-\sinh \theta \eta_{n}+\cosh \theta \zeta_{n}^{*},
\end{gathered}
$$

Equations (5.9) represent a one dimensional Bogoliubov transformation and it well known [10] that a vacuum state for $\eta_{n}, \zeta_{n}$ is given by $|\psi\rangle$ where

$$
|\psi\rangle=\exp \left[\left(a b-a^{*} b^{*}\right) \theta\right]|0\rangle \text {. }
$$

Now one can easily see from (5.7), (5.8) that $\eta_{j}, \zeta_{j}, 1 \leqq j \leqq n-1$, commute with $a, b$, $a^{*}, b^{*}$. Hence $|\psi\rangle$ as defined in (5.10) is also a vacuum for $\eta_{j}, \zeta_{j}, 1 \leqq j \leqq n-1$. Thus $|\psi\rangle$ is the state we are looking for.

We wish to construct the wave functions for Theorem 5.1 out of the states $|\psi\rangle$ of (5.10). There are however two problems to be solved. The first is that if we take the $a_{i}, b_{i}$ to be operators $a_{k}, a_{-k}$ with $k \in Z^{3}$ then $|\psi\rangle$ in (5.10) satisfies periodic boundary conditions on $\Lambda_{N}$ instead of Dirichlet. This problem may be rectified by taking the $a_{i}, b_{i}$ to be annihilation operators corresponding to products of sine functions. The second problem is that $|\psi\rangle$ does not have a fixed particle number. We solve this by employing a trick which goes back to [8].

We turn to the first problem. Let $d$ be a Boson operator so $\left[d, d^{*}\right]=1$ and consider the quadratic expression

$$
2(1+\beta) d^{*} d+d^{2}+d^{* 2}
$$

We can calculate the ground state energy of (5.11) by making a Bogoliubov transformation

$$
d=\cosh \theta \eta-\sinh \theta \eta^{*} .
$$

The expression (5.11) is given in terms of $\eta$ by

$$
\begin{aligned}
& {[2(1+\beta) \cosh 2 \theta-2 \sinh 2 \theta] \eta^{*} \eta+[\cosh 2 \theta-(1+\beta) \sinh 2 \theta]\left[\eta^{2}+\eta^{* 2}\right]} \\
& \quad+2(1+\beta) \sinh ^{2} \theta-\sinh 2 \theta .
\end{aligned}
$$

If we take $\theta$ to be given by (5.5) then it is evident that the ground state of (5.11) is a vacuum of $\eta$ and the value of (5.11) on the ground state is $\left(\beta^{2}+2 \beta\right)^{1 / 2}-1-\beta$. 
We calculate the vacuum of $\eta$ in terms of the vacuum $|0\rangle$ of $d$ by following the well known method. We write $\eta$ in (5.12) as a function of $\theta, \eta=\eta(\theta)$, and look for a unitary operator $U(\theta)$ such that

$$
\eta(\theta)=U(\theta) d U(\theta)^{*},
$$

in which case it follows that the vacuum $|\psi\rangle$ for $\eta(\theta)$ is given by

$$
|\psi\rangle=U(\theta)|0\rangle \text {. }
$$

It is clear from (5.12) that the $U(\theta)$ form a group and hence

$$
U(\theta)=\exp [L \theta],
$$

for some anti-symmetric operator $L$. On differentiating (5.14) with respect to $\theta$ and using (5.12), (5.16) we find that $L$ and $d$ are related by

$$
d^{*}=[L, d],
$$

and so we may take

$$
L=\frac{1}{2}\left[d^{2}-d^{* 2}\right] \text {. }
$$

It follows then that

$$
|\psi\rangle=\exp \left[\frac{1}{2}\left(d^{2}-d^{* 2}\right) \theta\right]|0\rangle
$$

with $\theta$ given by (5.5).

For $k=\left(k_{1}, k_{2}, k_{3}\right)$ in $Z^{3}$ let $\|k\|$ and $\operatorname{sgn} k$ be defined by

$$
\|k\|=\left(\left|k_{1}\right|,\left|k_{2}\right|,\left|k_{3}\right|\right) ; \quad \operatorname{sgn} k=\prod_{i=1}^{3} \operatorname{sgn} k_{i} .
$$

We shall denote by $k>0$ if each $k_{i}>0, i=1,2,3$. If $k \in Z^{3}$ with $k>0$, let $g_{k}$ be the annihilation operator corresponding to the wave function

$$
(2 / L)^{3 / 2} \sin \left(2 \pi k_{1} x_{1} / L\right) \sin \left(2 \pi k_{2} x_{2} / L\right) \sin \left(2 \pi k_{3} x_{3} / L\right)
$$

on the box $\Lambda_{L}$ with side of length $L$. We shall construct the wave function for Theorem 5.1 out of the operators $g_{k}$.

Let $B_{\gamma}$ be the set of $k \in Z^{3}$ with $k>0$ and $|k| \leqq \gamma$. For $\gamma \geqq \sqrt{3}, B_{\gamma}$ is nonempty with say $n(\gamma)$ elements. We define $h_{0}$ by

$$
h_{0}=\left(\sum_{k \in B_{\gamma}} g_{k}\right) / n(\gamma)^{1 / 2}
$$

Let $\delta_{\gamma}=[4 \gamma]+1$, where [] denotes integer part, and $L_{\gamma}$ be the lattice of vectors in $Z^{3}$ of the form $\left(\left(2 m_{1}+1\right) \delta_{\gamma},\left(2 m_{2}+1\right) \delta_{\gamma},\left(2 m_{3}+1\right) \delta_{\gamma}\right)$, where the $m_{i}, i=1,2,3$ are integers. For $k \in L_{\gamma}$ with $k>0$ we define $h_{k}$ to be

$$
h_{k}=\left(\sum_{m \in W_{\gamma}}(\operatorname{sgn} m) g_{m+k}\right) / 2 \sqrt{2} n(\gamma)^{1 / 2},
$$

where $W_{\gamma}$ is the set of $m \in Z^{3}$ with $\|m\| \in B_{\gamma}$.

Let $u=\left(h_{0} h_{0}^{*}\right)^{-1 / 2} h_{0}$. It is evident that $u u^{*}=1$ but $u^{*} u=1$ only on wave functions which have zero projection on the 0 eigenspace of $h_{0}^{*} h_{0}$. For $k \in L_{\gamma}$ we put $c_{k}=u^{*} h_{k}$, and it is clear that the $c_{k}$ almost satisfy canonical commutation relations when there are a large number of $h_{0}$ particles. 
Let $d_{k}, k \in L_{\gamma}$, be a set of Boson operators satisfying canonical commutation relations. For $T \geqq \delta_{\gamma}$ let $L_{\gamma, T}$ be the set of $k=\left(k_{1}, k_{2}, k_{3}\right) \in L_{\gamma}$ with $k>0$ and $\left|k_{i}\right| \leqq T$, $i=1,2,3$. If $|0\rangle$ is the vacuum of the $d_{k}, k \in L_{\gamma}$, we write $|\Psi\rangle$ as

$$
|\Psi\rangle=\exp \left[\sum_{k \in L_{\gamma}, T} \frac{1}{2}\left(d_{k}^{2}-d_{k}^{* 2}\right) \theta\right]|0\rangle
$$

Now (5.23) may be rewritten as

$$
|\Psi\rangle=\lim _{j \rightarrow \infty} P_{j}\left(\theta ; d_{k}^{*}, k \in L_{\gamma, T}\right)|0\rangle,
$$

where the $P_{j}$ is a polynomial of degree $j$ in the $d_{k}^{*}, k \in L_{\gamma, T}$, corresponding to the projection of $\Psi$ onto the space

$$
\sum_{k \in L_{\gamma}, T} d_{k}^{*} d_{k} \leqq j
$$

We can clearly write $\Psi=\Psi_{1}+\Psi_{2}$, where

$$
\left|\Psi_{1}\right\rangle=P_{N-5}\left(\theta ; d_{k}^{*}, k \in L_{\gamma, T}\right)|0\rangle
$$

and $\Psi_{2}$ is orthogonal to $\Psi_{1}$.

We may now define the wave functions for Theorem 5.1. Let $\psi_{\gamma, T, \beta}$ be the normalized state parallel to

$$
P_{N-5}\left(\theta ; c_{k}^{*}, k \in L_{\gamma, T}\right) \frac{\left(h_{0}^{*}\right)^{N}}{[N !]^{1 / 2}}|0\rangle,
$$

where $|0\rangle$ is the vacuum of the operators $g_{k}, k \in Z^{3}, k>0$. Observe that (5.27) is an $N$ particle state. The key fact which we shall use to estimate the kinetic and potential energy of (5.27) is the following: let $q\left(c_{k}, c_{k}^{*} ; k \in L_{\gamma, T}\right)$ be a polynomial in $c_{k}, c_{k}^{*}$ of degree less than 5 . Then

$$
\left\langle\psi_{\gamma, T, \beta}|q| \psi_{\gamma, T, \beta}\right\rangle=\left\langle\Psi_{1}\left|q\left(d_{k}, d_{k}^{*} ; k \in L_{\gamma, T}\right)\right| \Psi_{1}\right\rangle /\left\langle\Psi_{1} \mid \Psi_{1}\right\rangle .
$$

Lemma 5.2. Let $K_{N}$ be given by (1.8). Suppose $0<\beta \leqq 1, \gamma>\sqrt{3}$ and $\gamma, T, \beta, N$ satisfy the inequality

$$
T^{3}<\varepsilon \gamma^{3} \beta^{1 / 2} N
$$

for sufficiently small universal constant $\varepsilon>0$. Then there is a universal constant $C$ such that

$$
\left\langle\psi_{\gamma, T, \beta}\left|K_{N}\right| \psi_{\gamma, T, \beta}\right\rangle \leqq C\left[\frac{N \gamma^{2}}{L^{2}}+\frac{T}{\gamma^{3} L^{2} \beta^{1 / 2}}\right] .
$$

Proof. First observe that

$$
\begin{aligned}
& \left\langle\psi_{\gamma, T, \beta}\left|K_{N}\right| \psi_{\gamma, T, \beta}\right\rangle \\
& \quad=\frac{\mathbf{4} \pi^{2}}{L^{2}} \sum_{k \in \beta_{\gamma}} k^{2}\left\langle\psi\left|g_{k}^{*} g_{k}\right| \psi\right\rangle+\frac{4 \pi^{2}}{L^{2}} \sum_{m \in W_{\gamma}, k \in L_{\gamma, T}}(m+k)^{2}\left\langle\psi\left|g_{m+k}^{*} g_{m+k}\right| \psi\right\rangle .
\end{aligned}
$$

Since $\psi=\psi_{\gamma, T, \beta}$ is an eigenfunction of the number operator

$$
n=h_{0}^{*} h_{0}+\sum_{k \in L_{\gamma}, T} h_{k}^{*} h_{k}
$$


with eigenvalue $N$, it follows that

$$
\left\langle\psi\left|h_{0}^{*} h_{0}\right| \psi\right\rangle \leqq N
$$

from whence we have

$$
\left\langle\psi\left|g_{k}^{*} g_{k}\right| \psi\right\rangle \leqq N / n(\gamma), \quad k \in \beta_{\gamma} .
$$

Thus the first sum on the right in (5.31) is bounded above by the first term on the right in (5.30).

To estimate the second sum on the right in (5.31) we use (5.28). It is clear that this sum is bounded above by

$$
\frac{C T^{2}}{L^{2}} \sum_{k \in L_{\gamma}, T}\left\langle\psi\left|c_{k}^{*} c_{k}\right| \psi\right\rangle,
$$

and we have from (5.28) the identity

$$
\left\langle\psi\left|c_{k}^{*} c_{k}\right| \psi\right\rangle=\left\langle\Psi_{1}\left|d_{k}^{*} d_{k}\right| \Psi_{1}\right\rangle /\left\langle\Psi_{1} \mid \Psi_{1}\right\rangle .
$$

It is a simple calculation to see that

$$
\left\langle\Psi\left|\sum_{k \in L_{\gamma}, T} d_{k}^{*} d_{k}\right| \Psi\right\rangle \leqq \frac{A T^{3}}{\gamma^{3} \beta^{1 / 2}},
$$

for some constant $A$, and hence by the Chebyshev inequality we conclude

$$
\left\langle\Psi_{2} \mid \Psi_{2}\right\rangle \leqq A T^{3} / \gamma^{3} \beta^{1 / 2}(N-5) .
$$

This if $\varepsilon$ in (5.29) is sufficiently small we have $\left\langle\Psi_{1} \mid \Psi_{1}\right\rangle \geqq 1 / 2$. Furthermore

$$
\left\langle\Psi_{1}\left|\sum_{k \in L_{\gamma}, T} d_{k}^{*} d_{k}\right| \Psi_{1}\right\rangle
$$

is bounded above by the left side of (5.37). Hence, on using this inequality we have that the second sum on the right in (5.31) is bounded above by the second sum in (5.30) for suitable universal constant $C$.

Next we turn to estimating the potential energy.

Lemma 5.3. Let $S_{\gamma}$ be the set of $k$ in $Z^{3}$ such that there exists $n \in Z^{3}$ with $n, n+k \in W_{\gamma}$. Suppose $A_{k}$ is defined by (2.6) and $\psi=\psi_{\gamma, T, \beta}$. Then the expression $\left\langle\psi\left|A_{k}\right| \psi\right\rangle$ is nonzero in 3 cases: (a) If $k \in S_{\gamma}$; (b) If $k=k_{1}+k_{2}$ with $k_{1} \in S_{\gamma}$ and $k_{2} \in L_{\gamma}$; (c) If $k=k_{1}$ $+k_{2}$ with $k_{1} \in S_{\gamma}$ and $k_{2}+\left(\delta_{\gamma}, \delta_{\gamma}, \delta_{\gamma}\right) \in L_{\gamma}$.

In (a) $\left\langle\psi\left|A_{k}\right| \psi\right\rangle$ is given by

$$
\left\langle\psi\left|A_{k}\right| \psi\right\rangle=\frac{N}{8 n(\gamma)} \sum_{m}(\operatorname{sgn} m) \operatorname{sgn}(m+k),
$$

where the sum with respect to $m$ is over all $m \in Z^{3}$ with $m, m+k \in W_{\gamma}$.

In (b) $\left\langle\psi\left|A_{k}\right| \psi\right\rangle$ is given by

$$
\begin{aligned}
\left\langle\psi\left|A_{k}\right| \psi\right\rangle= & \frac{\operatorname{sgn} k}{2^{9 / 2} n(\gamma)}\left\langle\psi\left|h_{0}^{*} h_{\left\|k_{2}\right\|}+h_{0} h_{\left\|k_{2}\right\|}^{*}\right| \psi\right\rangle \\
& \cdot \sum_{m}(\operatorname{sgn} m) \operatorname{sgn}\left[\|m+k\|-\left\|k_{2}\right\|\right],
\end{aligned}
$$


where the sum with respect to $m$ is over all $m \in Z^{3}$ with $m, m+k_{1} \in W_{\gamma}$.

In (c) $\left\langle\psi\left|A_{k}\right| \psi\right\rangle$ is given by

$$
\left\langle\psi\left|A_{k}\right| \psi\right\rangle=\sum_{m \in L_{\gamma}} \lambda_{m}\left\langle\psi\left|h_{\left\|m+k_{2}\right\|}^{*} h_{\|m\|}\right| \psi\right\rangle,
$$

where the $\lambda_{m}$ are real numbers satisfying $\left|\lambda_{m}\right| \leqq 1 / 8$.

Proof. If $\left\langle\psi\left|a_{n+k}^{*} a_{n}\right| \psi\right\rangle \neq 0$, we need to have $n-t \in W_{\gamma}, n+k-t^{\prime} \in W_{\gamma}$, where $t, t^{\prime}$ lie in the union of 0 and $L_{\gamma}$. If $t=t^{\prime}$ we are in case (a). Otherwise $t^{\prime}=t+k_{2}$ with $k_{2} \neq 0$. If either $t$ or $t^{\prime}$ is zero then $k_{2}$ must be in $L_{\gamma}$. This is case (b). Finally, if both $t, t^{\prime}$ are in $L_{\gamma}$ then we must have $k_{2}+\left(\delta_{\gamma}, \delta_{\gamma}, \delta_{\gamma}\right) \in L_{\gamma}$. This is case (c).

To get the expressions (5.40), (5.41), (5.42) observe that if $m \in W_{\gamma}$ then

$$
a_{m}|\psi\rangle=\frac{i}{2^{3 / 2}}(\operatorname{sgn} m) \frac{h_{0}}{n(\gamma)^{1 / 2}}|\psi\rangle .
$$

If $m$ is such that $m \in t+W_{\gamma}$, where $t \in L_{\gamma}$, then

$$
a_{m}|\psi\rangle=\frac{1}{2^{3 / 2}}(\operatorname{sgn} m)[\operatorname{sgn}(\|m\|-\|t\|)] \frac{h_{\|t\|}}{2^{3 / 2} n(\gamma)^{1 / 2}}|\psi\rangle .
$$

Now suppose $k \in S_{\gamma}$ and $n=n_{j}, j=1, \ldots, p$, are the elements $n$ with $n, n+k \in W_{\gamma}$. Then if $\left\langle\psi\left|a_{n+k}^{*} a_{n}\right| \psi\right\rangle \neq 0, n$ is of the form $n=n_{j}+m$ with $m \in L_{\gamma}$. In view of the fact that for $m \in L_{\gamma}$,

$$
\operatorname{sgn}\left[\left\|n_{j}+m\right\|-\|m\|\right] \operatorname{sgn}\left[\left\|n_{j}+k+m\right\|-\|m\|\right]=\left(\operatorname{sgn} n_{j}\right)\left(\operatorname{sgn} n_{j}+k\right),
$$

we have

$$
\left\langle\psi\left|A_{k}\right| \psi\right\rangle=\frac{1}{2^{3} n(\gamma)}\left\langle\psi\left|\sum_{m \in\{0\} \cup L_{\gamma}, T} h_{m}^{*} h_{m}\right| \psi\right\rangle \cdot \sum_{j=1}^{p}\left(\operatorname{sgn} n_{j}\right)\left(\operatorname{sgn} n_{j}+k\right),
$$

which gives (5.40).

Next we take case (b). Let $n=n_{j}, j=1, \ldots, p$ be the set of elements $n$ such that $n$, $n+k_{1} \in W_{\gamma}$. Then $\left\langle\psi\left|a_{n+k}^{*} a_{n}\right| \psi\right\rangle \neq 0$ only if $n=n_{j}$ or $n+k=-n_{j}$. Summing up these terms yields (5.41).

For the final case (c) let $n=n_{j}, j=1, \ldots, p$, be again the set of elements $n$ with $n$, $n+k_{1} \in W_{\gamma}$. Then for $\left\langle\psi\left|a_{n+k}^{*} a_{n}\right| \psi\right\rangle \neq 0$ we need $n=n_{j}+m$ with $m \in L_{\gamma}$. The result (5.42) follows from (5.43), (5.44) and the fact that $p \leqq 8 n(\gamma)$. Q.E.D.

Lemma 5.4. Let $J_{k}(\psi)$ be defined by (2.10). Then for $\psi=\psi_{\gamma, T, \beta}$ we have in case (a)

$$
J_{k}(\psi)+\left|\left\langle\psi\left|A_{k}\right| \psi\right\rangle\right|^{2}=\left(N^{2}-N\right)\left[\frac{1}{8 n(\gamma)} \sum_{m}(\operatorname{sgn} m) \operatorname{sgn}(m+k)\right]^{2} .
$$

In case (b) there is

$$
\begin{aligned}
J_{k}(\psi)+\left|\left\langle\psi\left|A_{k}\right| \psi\right\rangle\right|^{2}= & {\left[\left\langle\psi\left|\left(h_{0}^{*} h_{\left\|k_{2}\right\|}+h_{0} h_{\left\|k_{2}\right\|}^{*}\right)^{2}\right| \psi\right\rangle\right.} \\
& \left.-\left\langle\psi\left|h_{0}^{*} h_{0}+h_{\left\|k_{2}\right\|}^{*} h_{\left\|k_{2}\right\|}\right| \psi\right\rangle\right] \\
& \cdot\left[\frac{1}{2^{9 / 2} n(\gamma)} \sum_{m}(\operatorname{sgn} m) \operatorname{sgn}\left[\|m+k\|-\left\|k_{2}\right\|\right]\right]^{2} .
\end{aligned}
$$


In case (c) we have

$$
J_{k}(\psi)+\left|\left\langle\psi\left|A_{k}\right| \psi\right\rangle\right|^{2}=\left\langle\psi\left|B_{k}^{*} B_{k}\right| \psi\right\rangle-\sum_{m \in L_{\gamma, T}}\left\langle\psi\left|C_{k, m}^{*} C_{k, m}\right| \psi\right\rangle,
$$

where

$$
\begin{gathered}
B_{k}=\sum_{\|m\|,\left\|m+k_{2}\right\| \in L_{\gamma, T}} \lambda_{m} h_{\left\|m+k_{2}\right\|}^{*} h_{\|m\|}, \\
C_{k, m}=\sum_{\substack{\|n\|=m \\
\left\|n+k_{2}\right\| \in L_{\gamma, T}}} \lambda_{n} h_{\left\|n+k_{2}\right\|}, \quad m \in L_{\gamma, T} .
\end{gathered}
$$

Proof. Similar to Lemma 5.3.

Lemma 5.5. Suppose $\gamma, T, \beta, N$ satisfy the conditions of Lemma 5.2. Then for $\psi=\psi_{\gamma, T, \beta}$,

$$
\left\langle\psi\left|h_{0}^{*} h_{0}\right| \psi\right\rangle \geqq N / 2,
$$

for sufficiently large $N$, and for sufficiently large $N$ depending only on $\beta$ there is a universal constant $C$ such that

$$
\left\langle\psi\left|\left(h_{0}^{*} h_{k}+h_{0} h_{k}^{*}\right)^{2}\right| \psi\right\rangle \leqq C \beta^{1 / 2} N,
$$

for $k \in L_{\gamma, T}$.

Proof. The proof of (5.52) follows by an argument similar to that given in Lemma 5.2. We have

$$
\left\langle\psi\left|h_{0}^{*} h_{0}\right| \psi\right\rangle=N-\left\langle\psi\left|\sum_{k \in L_{\gamma, T}} h_{k}^{*} h_{k}\right| \psi\right\rangle=N-\left\langle\Psi_{1}\left|\sum_{k \in L_{\gamma, T}} d_{k}^{*} d_{k}\right| \Psi_{1}\right\rangle /\left\langle\Psi_{1} \mid \Psi_{1}\right\rangle .
$$

If we choose $\varepsilon$ in (5.29) sufficiently small, then (5.52) follows from (5.37).

We turn to the proof of (5.53). It is easy to see that

$$
\left\langle\Psi\left|\left(d_{k}+d_{k}^{*}\right)^{2}\right| \Psi\right\rangle \leqq \beta^{1 / 2},
$$

and hence

$$
\left\langle\Psi_{1}\left|\left(d_{k}+d_{k}^{*}\right)^{2}\right| \Psi_{1}\right\rangle+2 \operatorname{Re}\left\langle\Psi_{1}\left|\left(d_{k}+d_{k}^{*}\right)^{2}\right| \Psi_{2}\right\rangle \leqq \beta^{1 / 2} .
$$

It is clear that

$$
\left\langle\Psi_{1}\left|\left(d_{k}+d_{k}^{*}\right)^{2}\right| \Psi_{2}\right\rangle=\left\langle\Psi_{1}\left|d_{k}^{2}+d_{k}^{* 2}\right| \Psi_{2}\right\rangle=2 \operatorname{Re}\left\langle\Psi_{1}\left|d_{k}^{* 2}\right| \Psi_{2}\right\rangle .
$$

Now we have

$$
\begin{aligned}
\left|\left\langle\Psi_{1}\left|d_{k}^{* 2}\right| \Psi_{2}\right\rangle\right| & \leqq\left\langle\Psi_{1}\left|d_{k}^{* 2} d_{k}^{2}\right| \Psi_{1}\right\rangle^{1 / 2}\left\langle\Psi_{2} \mid \Psi_{2}\right\rangle^{1 / 2} \\
& \leqq\left\langle\Psi\left|d_{k}^{* 2} d_{k}^{2}\right| \Psi\right\rangle^{1 / 2}\left\langle\Psi_{2} \mid \Psi_{2}\right\rangle^{1 / 2} \\
& \leqq\left\langle\Psi_{2} \mid \Psi_{2}\right\rangle^{1 / 2} / C \beta^{1 / 2}
\end{aligned}
$$

where $C$ is a universal constant. Hence if we choose $\varepsilon$ in (5.29) to be a small constant times $\beta^{2}$ then we may conclude that

$$
\left\langle\Psi_{1}\left|\left(d_{k}+d_{k}^{*}\right)^{2}\right| \Psi_{1}\right\rangle \leqq 2 \beta^{1 / 2},
$$

and hence from (5.28) that

$$
\left\langle\psi\left|\left(c_{k}+c_{k}^{*}\right)^{2}\right| \psi\right\rangle \leqq 4 \beta^{1 / 2} .
$$


It follows therefore that

$$
\left\langle\psi\left|n\left(c_{k}+c_{k}^{*}\right)^{2}\right| \psi\right\rangle \leqq 4 N \beta^{1 / 2},
$$

and hence that

$$
\left\langle\psi\left|\left(h_{0}^{*} h_{0}+h_{k}^{*} h_{k}\right)\left(c_{k}+c_{k}^{*}\right)^{2}\right| \psi\right\rangle \leqq 4 N \beta^{1 / 2} .
$$

We wish to compare the expression on the left in (5.62) with the expression on the left in (5.53). First observe that the left of (5.62) may be written as

$$
\begin{aligned}
& \left\langle\psi\left|h_{0}^{*} h_{0} h_{k} h_{k}^{*}+h_{0} h_{0}^{*} h_{k}^{*} h_{k}\right| \psi\right\rangle+2\left\langle\psi\left|\left(h_{k}^{*} h_{k}\right)^{2}\right| \psi\right\rangle \\
& \quad+\left\langle\psi\left|\left(h_{k}^{*} h_{k}\right)\left(c_{k}^{2}+c_{k}^{* 2}\right)\right| \psi\right\rangle+\left\langle\psi\left|\left(h_{0}^{*} h_{0}\right)\left(c_{k}^{2}+c_{k}^{* 2}\right)\right| \psi\right\rangle .
\end{aligned}
$$

We estimate the difference between the last term in (5.63) and $\langle\psi| h_{0}^{* 2} h_{k}^{2}$ $+h_{0}^{2} h_{k}^{* 2}|\psi\rangle$. Suppose we have

$$
h_{k}^{2}|\psi\rangle=\sum_{j=0}^{N-5} \alpha_{j}|N-j\rangle
$$

where $|N-j\rangle$ denotes a normalized eigenvector of $h_{0}^{*} h_{0}$ with eigenvalue $N-j$. Thus

$$
\sum_{j=0}^{N-5} \alpha_{j}^{2}=\left\langle\psi\left|h_{k}^{* 2} h_{k}^{2}\right| \psi\right\rangle=\left\langle\psi\left|c_{k}^{* 2} c_{k}^{2}\right| \psi\right\rangle=\left\langle\Psi_{1}\left|d_{k}^{* 2} d_{k}^{2}\right| \Psi_{1}\right\rangle /\left\langle\Psi_{1} \mid \Psi_{1}\right\rangle \leqq c / \beta,
$$

for some universal constant $C$. It is easy to see that

$$
\left.\left[h_{0}^{* 2} h_{k}^{2}-\left(h_{0}^{*} h_{0}\right) c_{k}^{2}\right] \psi\right\rangle=\sum_{j=0}^{N-5} \alpha_{j}\left\{1-\frac{(N+2-j)^{1 / 2}}{(N+1-j)^{1 / 2}}\right\} h_{0}^{* 2}|N-j\rangle .
$$

Hence we have

$$
\begin{aligned}
& \left\langle\left\langle\psi\left|h_{0}^{* 2} h_{k}^{2}-\left(h_{0}^{*} h_{0}\right) c_{k}^{2}\right| \psi\right\rangle\right. \\
& \quad \leqq\left[\sum_{j=0}^{N-5}(N+2-j)^{2} \alpha_{j}^{2}\left\{1-\frac{(N+2-j)^{1 / 2}}{(N+1-j)^{1 / 2}}\right\}^{2}\right]^{1 / 2} \leqq c / \beta^{1 / 2} \text { from (5.65). }
\end{aligned}
$$

The inequality (5.53) follows from (5.62), (5.63), and (5.67) Q.E.D.

Lemma 5.6. For $k$ in case (c) let $B_{k}$ be defined by (5.50). Suppose $T=N^{1 / 5} \gamma$. Then there is a universal constant $C$ such that

$$
\left\langle\psi\left|B_{k}^{*} B_{k}\right| \psi\right\rangle \leqq C N^{4 / 5} / \beta^{3 / 2} .
$$

Proof. It is clear that $\left\langle\psi\left|B_{k}^{*} B_{k}\right| \psi\right\rangle$ is a sum of terms of the form

$$
\left\langle\psi\left|c_{m}^{*} c_{n} c_{p}^{*} c_{q}\right| \psi\right\rangle
$$

with $m, n, p, q \in L_{\gamma, T}$, which is the same as

$$
\left\langle\Psi_{1}\left|d_{m}^{*} d_{n} d_{p}^{*} d_{q}\right| \Psi_{1}\right\rangle /\left\langle\Psi_{1} \mid \Psi_{1}\right\rangle .
$$

Now suppose that all the $m, n, p, q$ are different. Then one can see that

$$
\left\langle\Psi\left|d_{m}^{*} d_{n} d_{p}^{*} d_{q}\right| \Psi\right\rangle=0,
$$


from which it follows that

$$
\left\langle\Psi_{1}\left|d_{m}^{*} d_{n} d_{p}^{*} d_{q}\right| \Psi_{1}\right\rangle=-\left\langle\Psi_{2}\left|d_{m}^{*} d_{n} d_{p}^{*} d_{q}\right| \Psi_{2}\right\rangle .
$$

Hence we have

$$
\begin{aligned}
& \left\langle\Psi_{1} \mid \Psi_{1}\right\rangle\left|\sum_{m, n} \lambda_{m} \lambda_{n}\left\langle\psi\left|h_{\left\|n+k_{2}\right\|}^{*} h_{\|n\|} h_{\left\|m+k_{2}\right\|}^{*} h_{\|m\|}\right| \psi\right\rangle\right| \\
& =\left|\sum_{m, n} \lambda_{m} \lambda_{n}\left\langle\Psi_{2}\left|d_{\left\|n+k_{2}\right\|}^{*} d_{\|n\|} d_{\left\|m+k_{2}\right\|}^{*} d_{\|m\|}\right| \Psi_{2}\right\rangle\right| \\
& =\left|\sum_{m, n} \lambda_{m} \lambda_{n}\left\langle d_{\left\|n+k_{2}\right\|} d_{\left\|m+k_{2}\right\|} \Psi_{2} \mid d_{\|n\|} d_{\|m\|} \Psi_{2}\right\rangle\right| \\
& \leqq\left\langle\Psi_{2}\left|\left[\sum_{n \in L_{\gamma}, T} d_{n}^{*} d_{n}\right]^{2}\right| \Psi_{2}\right\rangle \leqq\left\langle\Psi\left|\left[\sum_{n \in L_{\gamma}, T} d_{n}^{*} d_{n}\right]^{3}\right| \Psi\right\rangle /(N-5),
\end{aligned}
$$

where the sum with respect to $m, n$ is over $m, n$ with $\left\|n+k_{2}\right\|,\|n\|,\left\|m+k_{2}\right\|,\|m\|$ all distinct. The last expression in (5.73) is bounded by a constant times $\left(T^{3} / \gamma^{3} \beta^{1 / 2}\right)^{3} / N$, which in turn is bounded by the right side of (5.68).

The terms in $B_{k}^{*} B_{k}$, where $\left\|n+k_{2}\right\|,\|n\|,\left\|m+k_{2}\right\|,\|m\|$ are not all distinct are still bounded by the last expression in (5.73), and hence we are finished. Q.E.D.

Next we obtain a Fourier representation for $|x-y|^{-1}$ as $x, y$ vary in $\Lambda_{L}$.

Lemma 5.7. For $x, y \in \Lambda_{L},|x-y|^{-1}$ has the representation

$$
|x-y|^{-1}=\sum_{4 k \in Z^{3}} v(k) e^{2 \pi i k \cdot(x-y) / L},
$$

and $v(k)$ is given by the formula

$$
v(k)=\frac{1}{64 \pi L|k|^{2}}[1-\cos |4 \pi k|]
$$

Proof. The maximum value of $|x-y|$ as $x, y$ vary in $\Lambda_{L}$ is $\sqrt{3} L$. Now let $f(x)$ be the cut off Coulomb potential

$$
f(x)=|x|^{-1}, \quad|x|<2 L, \quad f(x)=0, \quad|x|>2 L .
$$

The function $f(x)$ can be represented in a cube with side of length $4 L$ by a Fourier series

$$
f(x)=\sum_{4 k \in Z^{3}} v(k) e^{2 \pi i k \cdot x / L}
$$

where $v(k)$ is given by

$$
(4 L)^{3} v(k)=\int_{|x|<2 L}|x|^{-1} e^{2 \pi i k \cdot x / L} d x .
$$

If we evaluate the integral in (5.78), we obtain (5.75) and so (5.74) Q.E.D.

Let $J_{k}(\psi)$ be the potential energy of $\psi=\psi_{\gamma, T, \beta}$ corresponding to $\exp [2 \pi i k$ $\cdot(x-y) / L]$. If $k$ is in $Z^{3}$, then $J_{k}(\psi)$ is defined just as in (2.10). We shall show that.

Lemma 5.8. Suppose $k=\left(k_{1}, k_{2}, k_{3}\right)$ is such that $k_{i} \notin Z, i=1,2,3$, but $4 k \in Z^{3}$. Let $\psi=\psi_{\gamma, T, \beta}$ with $T=N^{1 / 5} \gamma$. The $\beta$ can be chosen sufficiently small independent of $k$ 
and $N$ sufficiently large depending only on $\beta$ such that $J_{k}(\psi)<-C_{k} N$ for some $C_{k}>0$.

Proof. $J_{k}(\psi)$ can be written in the form

$$
\begin{aligned}
J_{k}(\psi)= & \left\langle\psi\left|A^{*} A\right| \psi\right\rangle-|\langle\psi|A| \psi\rangle|^{2}-a^{2} N-\sum_{n \in L_{\gamma, T}} \alpha_{n}^{2}\left\langle\psi\left|h_{0}^{*} h_{0}+h_{n}^{*} h_{n}\right| \psi\right\rangle \\
& -\sum_{n} \beta_{n}^{2} \sum_{m \in L_{\gamma, T}}\left\langle\psi\left|C_{n, m}^{*} C_{n, m}\right| \psi\right\rangle,
\end{aligned}
$$

where

$$
A=a_{n}+\sum_{n \in L_{\gamma}, T} \alpha_{n}\left(h_{0}^{*} h_{n}+h_{0} h_{n}^{*}\right)+\sum_{n} \beta_{n} B_{n},
$$

and $B_{n}, C_{n, m}$ are defined as in (5.50), (5.51). This follows by expanding exp[2 $2 \pi i k$ $\cdot x / L]$ as a Fourier series in $\exp [2 \pi i m \cdot x / L]$ with $m \in Z^{3}$. If we let $B=A-a_{n}$, it is clear that

$$
J_{k}(\psi) \leqq\left\langle\psi\left|B^{*} B\right| \psi\right\rangle-a^{2} N-\sum_{n \in L_{\gamma}, T} \alpha_{n}^{2}\left\langle\psi\left|h_{0}^{*} h_{0}\right| \psi\right\rangle .
$$

The result follows from the previous lemmas by observing that the $\alpha_{n}$ are about the same size as the $\beta_{n}$. Q.E.D.

The potential energy of $\psi=\psi_{\gamma, T, \beta}$ is given by

$$
\sum_{4 k \in Z^{3}} v(k) J_{k}(\psi) \text {. }
$$

It is easy to see from the previous lemmas that by choosing $T=N^{1 / 5} / \gamma$ and $\beta$ sufficiently small we have

$$
\sum_{4 k \in Z^{3}} v(k) J_{k}(\psi) \leqq \frac{-C T}{L} N,
$$

for some constant $C>0$. Theorem 5.1 now follows by using (5.83), Lemma 5.2, and arguing as at the end of Sect. 2 .

Acknowledgements. Most of the research reported here was carried out while I was on sabbatical leave at the Institute for Theoretical Physics of the University of Vienna. I should like to thank Prof. Thirring and the mathematical physics group in Vienna for the invitation and hospitality during my stay. Special thanks are due to Bernd Baumgartner for several helpful conversations.

Thanks are also due to Elliott Lieb for bringing my attention to [10] of the bibliography.

\section{References}

1. Bogoliubov, N.N.: On the theory of superfluidity. J. Phys. (USSR) 11, $23-32$ (1947)

2. Conlon, J.: The ground state energy of a classical gas. Commun. Math. Phys. 94, 439-458 (1984)

3. Dyson, F.: Ground state energy of a finite system of charged particles. J. Math. Phys. 8, 15381545 (1967)

4. Dyson, F., Lenard, A.: Stability of matter. I. J. Math. Phys. 8, 423-434 (1967)

5. Fefferman, C.: The uncertainty principle. Bull. Am. Math. Soc. 9, 129-206 (1983)

6. Foldy, L.: Charged boson gas. Phys. Rev. 124, 649-651 (1961)

7. Hoffman-Ostenhoff, M.T.: Schrödinger inequalities and asymptotic behavior of the electron density of atoms and molecules. Phys. Rev. A 16, 1782-1785 (1977) 
8. Kromminga, A., Bolsterli, M.: Perturbation theory of many boson systems. Phys. Rev. 128, 2887-2897 (1962)

9. Lieb, E.: Simplified approach to the ground state energy of an imperfect Bose gas. Phys. Rev. 130, 2518-2528 (1963)

10. Lieb, E.: The bose fluid, Lectures in theoretical physics, Vol. VIIC, pp. 175-224. Boulder: The University of Colorado Press, 1965

11. Reed, M., Simon, B.: Methods of modern mathematical physics. II. Fourier analysis, selfadjointness, New York: Academic Press 1975

Communicated by B. Simon

Received December 10, 1984; in revised form March 5, 1985 
